(C) 2022, The Authors. Published by Elsevier Inc. and Fass Inc. on behalf of the American Dairy Science Association ${ }^{\circledR}$. This is an open access article under the CC BY license (http://creativecommons.org/licenses/by/4.0/).

\title{
Invited review: A quarter of a century-International genetic evaluation of dairy sires using MACE methodology
}

\author{
M. A. Nilforooshan ${ }^{1 *}$ (이 and H. Jorjani ${ }^{2}$ (๑) \\ ${ }^{1}$ Livestock Improvement Corporation, Private Bag 3016, Hamilton 3240, New Zealand \\ ${ }^{2}$ Department of Animal Breeding and Genetics, Swedish University of Agricultural Sciences, Box 7023, 75007 Uppsala, Sweden
}

\section{ABSTRACT}

For the past few decades, the international exchange of genetic materials has accelerated. This acceleration has been more substantial for dairy cattle compared with other species. The industry faced the need to put international genetic evaluation (IGE) systems in place. The Interbull Centre has been conducting IGE for various dairy cattle breeds and traits. This study reviews the past and the current status of IGE for dairy cattle, emphasizing the most prominent and wellestablished method of IGE, namely multiple acrosscountry evaluation (MACE), and the challenges that should be addressed in the future of IGE. The first IGE methods were simple conversion equations. Only a limited number of common bulls between pairs of countries were considered. These bulls were a biased sample of highly selected animals, with their daughters under preferential treatment in the importing countries. Genetic relationships among animals were not considered either. The MACE method was the first IGE method based on mixed-model theory that could handle genotype by environment interaction $(\mathrm{G} \times \mathrm{E})$ between countries. The $\mathrm{G} \times \mathrm{E}$ between countries is handled by treating the same trait in different countries as different traits, with genetic correlations less than unity between the traits. The $\mathrm{G} \times \mathrm{E}$ between countries is not solely due to different genetic expressions in different environments (countries), but is also attributable to different units or ways of measuring the trait, data editing, and statistical approaches and models used in different countries. The MACE method also considers different genetic means, genetic groups for unknown parents, heterogeneous genetic and residual variances among countries, and heterogeneous residual variances (precision weights for observations) within countries. Other IGE methods that came after MACE are rooted in MACE. The genomic revolution of the industry created new needs and opportunities. However, an

Received June 27, 2021.

Accepted September 6, 2021

*Corresponding author: mohammad.nilforooshan@lic.co.nz unwanted aspect of it was genomic preselection bias. Genomic preselection causes directional information loss from pre-culled animals (bias) in statistical models for genetic and genomic evaluations, and preselected progeny of a mating are no longer a random sample of possible progeny from that mating. National genetic evaluations without genotypes are input to MACE, and biases in national evaluations are propagated internationally through MACE. Genomic preselection for the Holstein breed is a source of concern for introducing bias to MACE, especially when genomic preselection is practiced intensively in the population. However, MACE continues to be useful for other breeds, among other species, or for non-IGE purposes. Future methods will need to make optimum use of genomic information and be free of genomic preselection bias.

Key words: MACE, international genetic evaluations, dairy cattle, deregressed proof

\section{INTRODUCTION AND HISTORY}

Since the introduction of artificial insemination techniques and use of frozen semen, the exchange of genetic materials across countries has been accelerated. Artificial insemination and international genetic exchanges have been more successful for dairy cattle than for other species. Embryo transfer is another form of genetic exchange, which is mainly practiced to take further advantage of passing genes of superior females as dams of bulls. In the early 1970s, large-scale importation of North American Holstein-Friesians to Europe began (Zarnecki et al., 1991). The importing countries were interested to know how daughters of foreign bulls would perform in the local herds and environment. Also, genetic variation is the key to selection in a breeding program. Thus, it is beneficial for populations to have open breeding programs with regular addition of new genetic materials from other populations. The dilemma is how to identify which individuals from other populations will produce high-yielding progeny that are well-integrated in the climate of the importing country. In the 1970s, the United Nations Food and Agriculture Organization led experiments to discover how the 
progeny of bulls from different populations performed in an importing country. Genetic merits of daughters of bulls from 10 Holstein-Friesian strains were compared in Polish commercial farms. The results later reported by Stolzman et al. (1988) and Zarnecki et al. (1991) indicated that Holstein strains from the United States, Canada, and Israel, and the Friesian strain from New Zealand yielded more milk in Poland compared with European strains (Denmark, Netherlands, Poland, Sweden, the UK, and West Germany). Another study (Rozzi et al., 1990) on a multinational genetic evaluation (the United States, Canada, and Italy) also showed that the average genetic merit of imported sires to Canada and Italy (mainly from the United States) was higher than for domestic sires for milk and fat yield.

By contrast, the average genetic merit of Canadian sires imported to the United States was lower than for domestic sires in the United States, which indicated that sires were imported to the United States for reasons other than production traits (Rozzi et al., 1990). The publication of those results was followed by a surge in international exchange of dairy cattle genetic materials, in the form of frozen semen, where many countries imported semen mainly from North America. The increased genetic exchange between countries urged the need for international genetic evaluations (IGE), as importing countries were interested to know which bulls to import to their population and environment, and exporting countries were seeking markets for their genetic materials.

Genetic ties between countries are of key importance to IGE. Increased exchange of genetic materials among countries potentially increases genetic variation, by opening national populations to foreign genetic materials. However, in reality, it has worked in the opposite way, as the effective population sizes have decreased considerably, due to a relatively small sample of superior bulls being heavily used internationally. For example, the effective population size of the Canadian Holstein population was reported as only 115 animals in 2011 (Stachowicz et al., 2011). Later, in 2020, the effective population size of Canadian Holsteins and Jerseys were estimated in a range of 43 to 66 and 64 to 85 animals, respectively (Makanjuola et al., 2020). During the 1980s and 1990s, the percentage of Holstein-Friesian cows with North American Holstein genetics increased from $2 \%$ to $38 \%$ in New Zealand (Harris and Kolver, 2001). The global population of Brown Swiss cattle was estimated to have an effective population size of 204 (Worede et al., 2013). Given the decreasing trend, the current effective population sizes should be considered even lower.

Published results of different national genetic evaluations (NGE), whether expressed as EBV or ETA, are not directly comparable. The main across-country differences are in the genetic base and scale, heritability, and genotype by environment interaction $(\mathbf{G} \times \mathbf{E})$. The latter causes reranking of bulls across countries. The $\mathrm{G} \times \mathrm{E}$ is important in quantitative trait variation, maintenance of genetic variation, and evolution (Huang et al., 2020). It can be seen as a biological (different gene expressions in different environments) or a statistical (different scale or genetic variation in different environments) phenomenon, or a combination of both (trait definition). In the animal breeding context, examples of biological $\mathrm{G} \times \mathrm{E}$ are different genotype expressions in different climates and production systems, and examples of statistical $\mathrm{G} \times \mathrm{E}$ are interactions caused by different units of measurement, different methods and models of genetic evaluation, and different pedigree and other sources of information used in the genetic evaluation.

One major obstacle to IGE was the lack of shared international data. Usually, within-country breed associations or NGE centers possessed the national data, and sharing was limited by concerns about data privacy and control. A need existed for an independent international organization for receiving national data, performing and maintaining the highest possible standards of IGE. That led to the formation of Interbull in 1983, to study the prerequisites of IGE. Interbull became a permanent subcommittee of the International Committee for Animal Recording (established in March 1951; https:// www.icar.org) in 1988. Interbull Centre, the operational unit of Interbull, was founded in 1991 in Uppsala, Sweden, to perform IGE based on standard methodology for making across-country bull comparisons possible. In 1995, the first routine IGE was performed, using a method called multiple across-country evaluation (MACE) for production traits (milk, fat, and protein yields) of Holstein bulls from 9 countries. Over time, the number of participating trait groups, breeds, and populations increased. Table 1 presents the time frame of the first official release of IGE results for different trait groups. Presently, IGE by Interbull covers 7 trait groups, 6 breed groups, and more than 30 populations. After initiating IGE for production traits, researchers from other Interbull participating countries took the initiative of introducing new trait groups to IGE. Examples are conformation (Lohuis et al., 1996; Klei and Lawlor, 1998), longevity (van der Linde and de Jong, 2002), calving (Pasman and Reinhardt, 2002), fertility (Jorjani, 2005), and workability traits (Bagnato et al., 2007; Jakobsen et al., 2008). Some of the populations comprise more than one country (Denmark-FinlandSweden and Germany-Austria), with similar climate and production systems, high genetic correlations with each other, and a joint NGE. The overall number of 
Table 1. First release of routine international genetic evaluations by trait group (changes in the number of breeds and the participating populations over time can be found at https://interbull.org/ib/maceev _archive; accessed Mar. 22, 2021)

\begin{tabular}{lll}
\hline Trait group & First release & Breeds $^{1}$ \\
\hline Production & Feb. 1995 & HOL, AYR \\
Conformation & Aug. 1999 & HOL \\
Udder health & May 2001 & AYR, BSW, GUE, HOL, JER \\
Longevity & Nov. 2004 & HOL \\
Calving & Feb. 2005 & HOL \\
Female fertility & Feb. 2007 & HOL \\
Workability & Jan. 2009 & BSW, HOL, JER, RDC \\
\hline
\end{tabular}

${ }^{1} \mathrm{AYR}=$ Ayrshire (later included in RDC); BSW = Brown Swiss; GUE $=$ Guernsey; HOL = Holstein; JER = Jersey; RDC = Red Dairy Cattle (e.g., AYR and Nordic Red breeds).

country-breed-trait combinations is over 2,000. The current number of population traits in IGE for each trait group and breed are presented in Table 2 .

Initially, due to the lack of both available data for IGE and well-established methodology, country pair comparisons were performed using a simple linear regression model called conversion equation (CE). This method (endorsed by the International Dairy Federation, 1981) regresses sire proofs from the exporting country to the base and scale of sire proofs in the importing country:

$$
\mathrm{P}_{\mathrm{imp}}=a+b \mathrm{P}_{\mathrm{exp}},
$$

where $\mathrm{P}$ is the proof or predicted genetic merit in the form EBV or ETA, in the importing (imp) or exporting (exp) country, and $a$ and $b$ are the intercept and regression coefficients, respectively.

For each pair of countries, a CE is calculated based on common bulls (bulls with official proofs in the 2 countries). To ensure that CE were both current and accurate, Interbull recommended calculating them using proofs for bulls born in the last $10 \mathrm{yr}$, with a minimum reliability of $75 \%$, and based on daughters in a minimum of 20 herds. To calculate $\mathrm{CE}$ for a pair of countries, a minimum of 20 bulls was required (Jorjani et al., 2001). Later, some modifications to the regression equation were suggested, such as using deregressed proofs (DRP) (Goddard, 1985) or adjusting proofs by their reliability values (Wilmink et al., 1986). The CE were officially in use for IGE until the introduction of the MACE methodology (Schaeffer, 1994). The latter is still in use, and it is the main focus of this study. This study provides a review of the methods proposed and used for IGE, and an overview of the past, present, and future of IGE. Research has been conducted on different aspects of IGE for a few decades, and this study touches on the most important aspects.

\section{METHODS PRIOR TO MACE}

As mentioned in the previous section, $\mathrm{CE}$ was the first IGE method. Even with the improvements made to CE (Goddard, 1985; Wilmink et al., 1986), they became increasingly at odds with those NGE that used mixed-model methodology, which was becoming very popular after Henderson's seminal work (Henderson, 1976). Generally, CE had the following problems:

1. Limited amount of information (use only the bulls proven in both countries).

2. No accounting for relatedness among bulls.

3. No accounting for information from a third country (i.e., strengthened connectedness between 2 countries via a third country or link provider).

4. Preferential treatment for daughters of foreign bulls.

5. Genetic correlation of 1 and no reranking of bulls between countries.

6. Bulls proven in more than one country are highly selected and a biased representation of the national sire populations.

Table 2. Current (August 2021) number of population traits in international genetic evaluations per trait group and breed (https://interbull.org/ib/maceev_archive; accessed Aug. 17, 2021)

\begin{tabular}{|c|c|c|c|c|c|c|}
\hline \multirow[b]{2}{*}{ Trait group } & \multicolumn{6}{|c|}{ Breed $^{1}$} \\
\hline & BSW & GUE & HOL & JER & $\mathrm{RDC}$ & SIM \\
\hline Production & 33 & 15 & 87 & 36 & 45 & 36 \\
\hline Conformation & 233 & 74 & 466 & 177 & 182 & 0 \\
\hline Udder health & 20 & 5 & 58 & 20 & 27 & 22 \\
\hline Longevity & 10 & 5 & 21 & 9 & 12 & 5 \\
\hline Calving & 12 & 0 & 56 & 0 & 11 & 0 \\
\hline Female fertility & 38 & 18 & 75 & 34 & 44 & 0 \\
\hline Workability & 7 & 0 & 27 & 6 & 14 & 0 \\
\hline
\end{tabular}


Even after introducing MACE, CE were still calculated based on international proofs, as an IGE by-product to be temporarily used for young bulls not yet included in the MACE system, and for cows.

Schaeffer (1985) made the first suggestion for using the mixed-model methodology for across-country genetic evaluation. We refer to this first suggestion as multiple country evaluation (MCE), in which the genetic correlation of unity was assumed between the traits measured in different countries. The MCE was a single-trait sire model, where sire proofs from different countries were combined, and each sire received a single international proof for a given trait. The MCE considered base but not scale or variance differences among countries. Rozzi et al. (1990) and Banos et al. (1991) used MCE with modifications for the comparison of multinational dairy cattle populations. Modifications were mainly related to the use of DRP, rules for forming the phantom parent groups (PPG), and standardization of each country's data. In MCE, not only the across-country genetic correlations were unity, but the heritabilities were also considered equal, and no $\mathrm{G} \times \mathrm{E}$ was modeled. Schaeffer (1985) used national bull proofs as the dependent variable in the model. Alternatives are DRP and average daughter yield deviations (DYD). From here on, we refer to any sort of dependent variable as observations. The model was written in matrix notation as follows:

$$
\mathbf{y}=\mathbf{X c}+\mathbf{Z Q g}+\mathbf{Z s}+\mathbf{e}
$$

with the corresponding mixed-model equations:

$\left[\begin{array}{ccc}\mathbf{X}^{\prime} \mathbf{D}^{-1} \mathbf{X} & \mathbf{X}^{\prime} \mathbf{D}^{-1} \mathbf{Z} \mathbf{Q} & \mathbf{X}^{\prime} \mathbf{D}^{-1} \mathbf{Z} \\ \mathbf{Q}^{\prime} \mathbf{Z}^{\prime} \mathbf{D}^{-1} \mathbf{X} & \mathbf{Q}^{\prime} \mathbf{Z}^{\prime} \mathbf{D}^{-1} \mathbf{Z} \mathbf{Q} & \mathbf{Q}^{\prime} \mathbf{Z}^{\prime} \mathbf{D}^{-1} \mathbf{Z} \\ \mathbf{Z}^{\prime} \mathbf{D}^{-1} \mathbf{X} & \mathbf{Z}^{\prime} \mathbf{D}^{-1} \mathbf{Z} \mathbf{Q} & \mathbf{Z}^{\prime} \mathbf{D}^{-1} \mathbf{Z}+\mathbf{A}^{-1} k\end{array}\right]\left[\begin{array}{l}\hat{\mathbf{c}} \\ \hat{\mathbf{g}} \\ \hat{\mathbf{s}}\end{array}\right]=\left[\begin{array}{c}\mathbf{X}^{\prime} \mathbf{D}^{-1} \mathbf{y} \\ \mathbf{Q}^{\prime} \mathbf{Z}^{\prime} \mathbf{D}^{-1} \mathbf{y} \\ \mathbf{Z}^{\prime} \mathbf{D}^{-1} \mathbf{y}\end{array}\right]$,

where $\mathbf{y}, \mathbf{c}, \mathbf{g}, \mathbf{s}$, and $\mathbf{e}$ are the vectors of observations, country means, PPG, sire additive genetic effects, and random residuals, respectively. Vectors $\hat{\mathbf{c}}, \hat{\mathbf{g}}$, and $\hat{\mathbf{s}}$ are the estimates for $\mathbf{c}, \mathbf{g}$, and $\mathbf{s}$. Matrices $\mathbf{X}, \mathbf{Z}$, and $\mathbf{Q}$ relate countries to records, sires to records, and sires to PPG, respectively; $\mathbf{A}$ is the additive genetic relationship matrix among sires, $k$ is the variance ratio equal to $\sigma_{e}^{2} / \sigma_{s}^{2}=\left(4-h^{2}\right) / h^{2}$, where $\sigma_{e}^{2}$ and $\sigma_{s}^{2}$ are the residual and sire genetic variances, respectively; $h^{2}$ is the heritability of the trait, assumed to be the same in all countries; and $\operatorname{Var}(\mathbf{e})=\mathbf{D} \sigma_{e}^{2}$, where $\mathbf{D}$ is the matrix of weighting factors. For $t$ number of countries,

$$
\begin{aligned}
\mathbf{D}=\sum{ }^{+} \mathbf{D}_{i} & =\left[\begin{array}{cccc}
\mathbf{D}_{1} & \mathbf{0} & \cdots & \mathbf{0} \\
\mathbf{0} & \mathbf{D}_{2} & \cdots & \mathbf{0} \\
\vdots & \vdots & \ddots & \vdots \\
\mathbf{0} & \mathbf{0} & \cdots & \mathbf{D}_{t}
\end{array}\right] \\
\mathbf{D}_{i} & =\operatorname{diag}\left(1 / n_{i j}\right),
\end{aligned}
$$

where $n_{i j}$ is the number of daughters for the $j$ th bull in country $i$. In MCE, one solution exists per bull $\left(\hat{s}_{i}\right)$, and proofs of $n$ bulls in $t$ countries are calculated as $\left[(\mathbf{Q} \hat{\mathbf{g}}+\hat{\mathbf{s}}) \otimes \mathbf{1}_{t}\right]+\left(\mathbf{1}_{n} \otimes \hat{\mathbf{c}}\right)$.

It is possible to consider different heritabilities for different countries in MCE by modifying Eq. [3] as follows. First, divide both sides of the equations by $k$. Second, replace $\mathbf{D}^{-1} / k$ with $\mathbf{D}^{-1} \mathbf{K}^{-1}$, where

$$
\begin{aligned}
\mathbf{K}=\sum{ }^{+} \mathbf{K}_{i} & =\left[\begin{array}{cccc}
\mathbf{K}_{1} & \mathbf{0} & \cdots & \mathbf{0} \\
\mathbf{0} & \mathbf{K}_{2} & \cdots & \mathbf{0} \\
\vdots & \vdots & \ddots & \vdots \\
\mathbf{0} & \mathbf{0} & \cdots & \mathbf{K}_{t}
\end{array}\right] \\
\mathbf{K}_{i} & =\operatorname{diag}\left(k_{i}\right) .
\end{aligned}
$$

The benefits of MCE were

1. Simultaneous comparisons of sires across multiple countries.

2. Use of an international pedigree for considering genetic relatedness among sires.

3. Moderating possible bias in proofs of imported sires via the relationship matrix.

4. Making use of a considerably larger amount of information, not limited to common bulls between 2 countries, and not limited to 2 countries.

The shortcomings of MCE were

1. Unity genetic correlation between countries (no $\mathrm{G} \times \mathrm{E})$.

2. Same sire (genetic) and same residual variances for the countries.

3. Traits measured in different countries should be in the same unit; otherwise data from different countries should be scaled to the same unit before the analysis and scaled back to the original units after the analysis.

Schaeffer (1994) applied a multivariate expansion of the sire equations in $\mathrm{MCE}$, to evaluate sires by country, thereby extending MCE to MACE and allowing the across-country genetic correlations to be less than 
unity. Although MCE was not officially used for IGE in practice, it was the foundation for the officially used method MACE.

\section{MACE}

One of the early attempts after the implementation of $\mathrm{CE}$ and before the implementation of MACE was a collaboration combining bull evaluations from the United States and Canada (Wiggans et al., 1992). That method involved weighted combining of parent averages and progeny contributions, with bull contributions removed. Combined reliabilities were calculated from combined daughter equivalents, equal to the sum of daughter equivalents in each country, with parent average daughter equivalents subtracted (Wiggans et al., 1992). This method was complex and limited to a country pair. A unified evaluation for all country pairs and not limited to data from a single country pair was advantageous and convenient.

Schaeffer (1994) introduced the mixed-model methodology for MACE, multiple across-country evaluation, officially used for IGE to date. Generally, MACE was the multiple-trait form of MCE, where data from each country were considered as different traits. This multivariate approach allowed different heritabilities and genetic correlations less than unity among the countries. As a result, bulls received international proofs on the base and scale of each country participating in the analysis, and could be ranked differently in different countries, accounting for $\mathrm{G} \times \mathrm{E}$ effects. With the availability of enough information on environmental factors (especially if the environmental effects were directional or imposed by the researcher), reaction norms would be the choice to model $\mathrm{G} \times \mathrm{E}$ (e.g., Kolmodin et al., 2002). However, usually environmental effects are unknown and not directional. In these situations, $\mathrm{G} \times \mathrm{E}$ has traditionally been modeled by multiple-trait models, considering a trait's expression in different environments as correlated traits (Falconer, 1952). The benefits of MACE over MCE were

1. Considering $\mathrm{G} \times \mathrm{E}$ across countries by enabling genetic correlations less than 1, with different genetic variances and heritabilities.

1.1. Animals receive an evaluation per country.

1.2. Animals rank differently in different countries.

2. Animals are evaluated in different scales, and traits do not need to have the same definition and be measured in the same unit (no scaling and back-scaling required).

3. Reliability on the scale of each country increases by transferring information across all countries in a more optimal way. Within-country predictions are weighted and combined by genetic correlations via an international pedigree.

Even in the absence of biological $\mathrm{G} \times \mathrm{E}$, different NGE models, trait definitions, and units of measurement can yield non-unity genetic correlations between countries (statistical $\mathrm{G} \times \mathrm{E}$ ).

\section{Methodology}

The MACE method is a multiple-trait sire model in which the same traits, measured and evaluated from different countries and NGE systems, are considered as different traits internationally. Observations in country $i$ are modeled as

$$
\mathbf{y}_{i}=\mathbf{c}_{i} \mathbf{1}+\mathbf{Z}_{i} \mathbf{Q g}_{i}+\mathbf{Z}_{i} \mathbf{s}_{i}+\mathbf{e}_{i}
$$

The complete (multiple-county) model is written as Eq. [2], with the difference that it is a multi-trait model rather than a single-trait model. For $t$ countries,

$$
\begin{gathered}
\mathbf{y}=\left[\begin{array}{c}
\mathbf{y}_{1} \\
\vdots \\
\mathbf{y}_{t}
\end{array}\right] ; \\
\mathbf{X}=\left[\begin{array}{cccc}
\mathbf{1}_{n_{1}} & \mathbf{0} & \cdots & \mathbf{0} \\
\mathbf{0} & \mathbf{1}_{n_{2}} & \cdots & \mathbf{0} \\
\vdots & \vdots & \ddots & \vdots \\
\mathbf{0} & \mathbf{0} & \cdots & \mathbf{1}_{n_{t}}
\end{array}\right] ; \\
\mathbf{Z}=\left[\begin{array}{cccc}
\mathbf{Z}_{1} & \mathbf{0} & \cdots & \mathbf{0} \\
\mathbf{0} & \mathbf{Z}_{2} & \cdots & \mathbf{0} \\
\vdots & \vdots & \ddots & \vdots \\
\mathbf{0} & \mathbf{0} & \cdots & \mathbf{Z}_{t}
\end{array}\right]
\end{gathered}
$$

In matrix notation, MACE is written as follows:

$$
\begin{aligned}
& {\left[\begin{array}{ccc}
\mathbf{X}^{\prime} \mathbf{D}^{-1} \mathbf{X} & \mathbf{0} & \mathbf{X}^{\prime} \mathbf{D}^{-1} \mathbf{Z} \\
\mathbf{0} & \mathbf{Q}^{\prime} \mathbf{A}^{-1} \mathbf{Q} \otimes \mathbf{G}^{-1} & -\mathbf{Q}^{\prime} \mathbf{A}^{-1} \otimes \mathbf{G}^{-1} \\
\mathbf{Z}^{\prime} \mathbf{D}^{-1} \mathbf{X} & -\mathbf{A}^{-1} \mathbf{Q} \otimes \mathbf{G}^{-1} & \mathbf{Z}^{\prime} \mathbf{D}^{-1} \mathbf{Z}+\mathbf{A}^{-1} \otimes \mathbf{G}^{-1}
\end{array}\right]\left[\begin{array}{c}
\hat{\mathbf{c}} \\
\hat{\mathbf{g}} \\
\mathbf{Q} \hat{\mathbf{g}}+\hat{\mathbf{s}}
\end{array}\right]} \\
& =\left[\begin{array}{c}
\mathbf{X}^{\prime} \mathbf{D}^{-1} \mathbf{y} \\
\mathbf{0} \\
\mathbf{Z}^{\prime} \mathbf{D}^{-1} \mathbf{y}
\end{array}\right]
\end{aligned}
$$

with the following statistical properties: 


$$
\left[\begin{array}{l}
\mathbf{y} \\
\mathbf{s} \\
\mathbf{e}
\end{array}\right]=\operatorname{MVN}\left[\left(\begin{array}{c}
\mathbf{X c}+\mathbf{Z Q g} \\
\mathbf{0} \\
\mathbf{0}
\end{array}\right),\left(\begin{array}{ccc}
\mathbf{Z}(\mathbf{A} \otimes \mathbf{G}) \mathbf{Z}^{\prime}+\mathbf{D} & (\mathbf{A} \otimes \mathbf{G}) \mathbf{Z}^{\prime} & \mathbf{D} \\
\mathbf{Z}(\mathbf{A} \otimes \mathbf{G}) & \mathbf{A} \otimes \mathbf{G} & \mathbf{0} \\
\mathbf{D} & \mathbf{0} & \mathbf{D}
\end{array}\right)\right],
$$

where $\mathbf{G}$ is the $t \times t$ sire additive genetic variance-covariance matrix between the countries. Considering $p_{i}$ being the number of observations in country $i$, and $n$ being the total number of sires in the pedigree, vectors $\mathbf{y}$ and $\mathbf{e}$ are $\Sigma p_{i} \times 1$, vector $\mathbf{s}$ is $(n \times t) \times 1$, matrix $\mathbf{D}$ is $\Sigma p_{i} \times \Sigma p_{i}$, matrix $\mathbf{A}$ is $n \times n$, matrix $\mathbf{Z}$ is $\Sigma p_{i} \times(n$ $\times t$ ), and MVN indicates multivariate normal. Similar to MCE, $\mathbf{D}$ has diagonal matrices $\mathbf{D}_{i}$ on its diagonals. Unlike MCE, $\mathbf{D}_{i}=\operatorname{diag}\left(\sigma_{e_{i}}^{2} / n_{i j}\right)$. Therefore, observations within and across traits (countries) are treated as residually independent, and both within- and acrosscountry heterogeneity of residual variances are modeled. To comply with the prerequisite of residually independent traits, only one trait per country is allowed in MACE, and it is assumed that daughter groups, by country, are distinct from one another. The withincountry observations (DRP or DYD) are residually uncorrelated because the bulls do not share daughters. However, this can be statistically violated if the NGE model or the pedigree information fails to adequately correct for genetic merits of mates or preferential treatment of daughters or ignores common effects between groups of daughters from different sires. Incorrect calculation of pseudo-observations (DRP or DYD) can also lead to residually correlated observations. If within-country observations were residually correlated, one of MACE's assumptions is not entirely fulfilled. Ignoring such covariances, if they exist, could instead result in greater prediction error variances than biased IGE (Schaeffer, 1994).

Matrix $\mathbf{A}$ is the additive genetic relationship matrix, which was initially among all proven sires and their male ancestors in the international pedigree. In 2013, based on a report by Jakobsen and Dürr (2012), matrix A was changed to include sire and dam relationships, instead of restricting to only the male ancestors via sires and maternal grandsires (MGS). Changing the MACE pedigree from sire-MGS to sire-dam was first proposed by de Jong (2003). The reasons were that in MACE with a sire-MGS pedigree, all bulls were directly connected to a PPG for the genetic contribution of their maternal granddam (MGD-PPG), and the genetic differences from parent average for each dam (Mendelian Sampling of the dam) were not being directly modeled. The effect of MGD-PPG on the scale of each country was large and undesirable, as it increased the differences between NGE and IGE more than necessary and in a suboptimal way. All MGD are unknown in sire-MGS MACE and are assigned to a PPG (Schaeffer, 1994). For each country included, separate PPG were used for sires, MGS, and MGD, as recommended by (Schaeffer, 1994), due to different selection intensities that might have been applied to each group. By adding dam information to the pedigree, PPG contributions were reduced and moved further (more generations) away from the proven bulls, and the suboptimal use of a same average MGD contribution for many different sires was substantially reduced. Because most bull dams had more than one proven son, including dams in the pedigree made important distinctions among son groups of different dams (de Jong, 2003). Including dam in the MACE pedigree improved the consistency between NGE and IGE, as dam information was generally included in the pedigree for NGE systems. Ignoring inbreeding coefficients in the population, the contributions of each bull to $\mathbf{A}^{-1}$ in the sire-MGS application were as shown below (Schaeffer, 1994):

$\begin{array}{ccccc} & \text { Bull } & \text { Sire } & \text { MGS } & \text { MGD } \\ \text { Bull } & x & -x / 2 & -x / 4 & -x / 4 \\ \text { Sire } & -x / 2 & x / 4 & x / 8 & x / 8 \\ \text { MGS } & -x / 4 & x / 8 & x / 16 & x / 16 \\ \text { MGD } & -x / 4 & x / 8 & x / 16 & x / 16\end{array}$

where $x$ is the additive genetic to Mendelian Sampling variance ratio (Mrode, 2005) equal to $16 /(m+11$ ), $m=0$ if both sire and MGS are known, $m=1$ if the sire is known and MGS is unknown, $m=4$ if the sire is unknown and MGS is known, and $m=5$ if both sire and MGS are unknown.

After changing to sire-dam pedigree, the contributions of animals to $\mathbf{A}^{-1}$ were as follows:

$$
\begin{array}{cccc} 
& \text { Bull } & \text { Sire } & \text { Dam } \\
\text { Bull } & x_{i i} & -x_{i s} / 2 & -x_{i d} / 2 \\
\text { Sire } & -x_{s i} / 2 & x_{s s} / 4 & x_{s d} / 4 \\
\text { Dam } & -x_{d i} / 2 & x_{d s} / 4 & x_{d d} / 4
\end{array}
$$

If both parents are known, all $x$ values are equal to $4 /(2$ $-F_{s}-F_{d}$ ), where $F_{s}$ and $F_{d}$ are inbreeding coefficients of the sire $(s)$ and dam $(d)$. If only one parent ( $p$ being either sire or dam) is known, then $x_{i i}=x_{i p}=x_{p i}=x_{p p}=$ $4 /\left(3-F_{p}\right)$, and the other $x$ values are equal to 0 , and if both parents are unknown, then $x_{i i}=1$, and the other $x$ values are equal to 0 .

The inputs for MACE are national observations (DRP or DYD), national residual weighting factors (total or effective number of daughter contributions), the $\mathbf{A}^{-1}$ 
matrix made from an international pedigree for bulls with official national proofs, and appended rows and columns corresponding to PPG, heritabilities reported by the countries, and sire variances and covariances.

\section{Country Sub-Setting}

Interbull estimates sire (genetic) variances and covariances. The residual variances are calculated based on the heritabilities reported by the countries and the corresponding sire variances. Due to computational challenges, genetic variances and covariances cannot be estimated simultaneously. The procedure used is to create a group of 3 to 4 countries with large populations and strong connectedness to all other countries (a link-provider group). The remaining countries, which are often smaller populations, are divided into subsets of 3 to 5 countries with relatively good connectedness among themselves (weak-link groups). Then, the genetic covariances are estimated for the link-provider group and 2 weak-link groups in turn. This procedure is continued until all genetic covariances are estimated. One consequence is that the genetic correlations among countries of a group are estimated several times, which need to be averaged. The resulting genetic variances and covariances among all countries are assembled into one genetic covariance matrix, which is then subjected to a bending procedure (Jorjani et al., 2003), to guarantee that a positive-definite matrix is always used in the IGE.

\section{Observations}

Observations are introduced via vector y. For several reasons, national sire proofs cannot be directly considered in the IGE: (1) national sire proofs are already regressed statistics; the existing genetic and residual dependencies between individual daughter phenotypes are taken into account in the NGE model and cannot be further taken into account in another model; (2) using national sire proofs in another genetic evaluation model double-counts the relationship coefficients among sires; (3) parameters such as heritability and the variance components for daughter phenotypes are not directly applicable to sire proofs; (4) national sire proofs are on a different base and scale than the daughter phenotypes for many traits of interest. For IGE, a pseudo-phenotype, which approximates a daughter average, is used for each sire and trait. The application of these pseudo-phenotypes is not limited to IGE, as they can also be used for GWAS (Guo et al., 2012) and for genomic NGE (Garrick et al., 2009). The pseudophenotypes come in the 2 forms of DYD and DRP, and should in either case be free from effects other than the genetic contributions of sires to the progeny phenotypes. The DYD are based on a concept first presented by VanRaden and Wiggans (1991), who defined DYD as a weighted average of daughter yield deviations (daughter yields deviated from solutions for known fixed effects and permanent environmental effects) adjusted for the merits of a bull's mates (dams of the daughters). For IGE, the use of DRP has been preferred over DYD (Sigurdsson and Banos, 1995). According to Sullivan (2020), an important advantage of using DRP in MACE is the reversibility of MACE results to national proofs, if the only information provided was from the national evaluation. Reversibility in this context means that if DRP were used in a MACE analysis for a single country, effectively excluding all foreign data from the MACE equations for that country, then the international MACE proofs would be identical to NGE proofs. In the application of MACE, sires who do have new information available from foreign countries will have MACE results that can differ from NGE, whereas sires with no new information (i.e., all information, including from relatives, contributed by only one country) will have MACE proofs exactly equal to NGE.

In deregression, all the information included in the NGE is preserved in DRP (Mark et al., 2002; Sullivan, 2020). The aim of deregression is to adjust the NGE proofs, by removing all effects in common between the NGE and IGE models, so that these effects can be reapplied to the DRP in MACE without double-counting the same information. The model used to generate DRP for MACE must be identical to the MACE model for this reversibility to work properly in terms of factors included (country + sire) and pedigree relationships and genetic groups used (i.e., the same $\mathbf{A}$ and $\mathbf{Q}$ matrices are used for deregression and in the MACE model). The DRP can include additive genetic contributions other than those of the sire, if these contributions are estimated at the national level but not within the MACE model. For example, any pedigree contributions that are not in common between the national and international pedigree (e.g., a different $\mathbf{A}$ matrix used for NGE versus MACE), which would mainly be contributions from the maternal pedigree (Mrode, 2005), are captured within the DRP and retained as an integrated part of both the NGE and the MACE results. The idea is to retain this additional information in a way that is consistent with how it was captured as a part of the NGE sire proofs. Deregression of national proofs for MACE is performed one country-trait at a time, because traits are analyzed independently in different countries. Consequently, MACE equations for a single country-trait (i) are involved: 


$\left[\begin{array}{cccc}\mathbf{1}^{\prime} \mathbf{R}_{i}^{-1} \mathbf{1} & \mathbf{1}^{\prime} \mathbf{R}_{i}^{-1} & \mathbf{0} & \mathbf{0} \\ \mathbf{R}_{i}^{-1} \mathbf{1} & \mathbf{R}_{i}^{-1}+\mathbf{A}^{s s} k_{i} & \mathbf{A}^{s p} k_{i} & \mathbf{A}^{s g} k_{i} \\ \mathbf{0} & \mathbf{A}^{p s} k_{i} & \mathbf{A}^{p p} k_{i} & \mathbf{A}^{p g} k_{i} \\ \mathbf{0} & \mathbf{A}^{g s} k_{i} & \mathbf{A}^{g p} k_{i} & \mathbf{A}^{\mathrm{gg}} k_{i}\end{array}\right]\left[\begin{array}{c}c_{i} \\ \mathbf{Q g}_{i}+\mathbf{s}_{i} \\ \mathbf{p}_{i} \\ \mathbf{g}_{i}\end{array}\right]=\left[\begin{array}{c}\mathbf{1}^{\prime} \mathbf{R}_{i}^{-1} \mathbf{y}_{i} \\ \mathbf{R}_{i}^{-1} \mathbf{y}_{i} \\ \mathbf{0} \\ \mathbf{0}\end{array}\right]$,

where $s, p$, and $g$ correspond to sires with proof, relatives without proof, and PPG in country $i$, making blocks of $\mathbf{A}^{-1}$ such as $\mathbf{A}^{s p}$, and $\mathbf{R}_{i}=\mathbf{D}_{i} / \sigma_{e_{i}}^{2}=\operatorname{diag}\left(1 / n_{i j}\right)$. One of the methods often used for deregression of national proofs was proposed by Jairath et al. (1998), which Interbull also uses for the incoming data to MACE. The method iteratively solves the above equations for $\mathbf{R}_{i}^{-1} \mathbf{y}_{i}$, and $\mathbf{y}_{i}=\mathbf{R}_{i}\left(\mathbf{R}_{i}^{-1} \mathbf{y}_{i}\right)$. The method is briefly presented in Appendix 1.

\section{Effective Daughter Contributions}

In a simple regression procedure, whenever the assumption of constant residual variance across observations (homoscedasticity) is violated (heteroscedasticity), a weighted regression model is applied to weight observations (inversely related to their residual variance) to minimize the sum of (weighted) squared residuals. This is the special case where observations are residually uncorrelated, but different accuracies are involved with different observations. The MACE method is also a weighted linear model in which heteroscedasticity is modeled within and across countries. For the first few years after the introduction of MACE, the reciprocal of the number of daughters involved in the evaluation of the bull was considered as the weighting factor in MACE and deregression (i.e., the greater the number of daughters, the higher the reliability and the less the uncertainty associated with the observation). Nevertheless, the residual weighting factors should be consistent with prediction error variances using progeny data in the NGE (Garrick et al., 2009). The number of daughters may not precisely represent the prediction error variance or the amount of daughter contribution to a sire's evaluation.

Fikse and Banos (2001) tested 6 other alternatives to the total number of daughters, called effective daughter contribution (EDC). Using the number of daughters overestimated the genetic variance. International reliabilities were affected by the choice of weighting factors, but the effect on international breeding values was marginal. The impact of weighting factors would be greater when different countries apply different NGE models and when genetic ties between some populations are weak (Fikse and Banos, 2001). The NGE model (whether it is a multiple lactation, repeatability, or any other model) should be considered in the choice of a proper weighting factor (EDC). Factors such as contemporary group structure, correlation between repeated records, and reliability of dams of daughters should be considered in the EDC calculation (Fikse and Banos, 2001).

Although the reliability of the national proof may be seen as a candidate for a weighting factor, it requires that countries predict reliabilities in the same way. A simulation study (Fikse and Sullivan, 1999) showed that weighting factors based on national reliabilities might introduce error to IGE.

\section{Phantom Parent Groups}

Like any form of BLUP, MACE regresses genetic merit solutions toward the population mean, which is assumed to be zero. As a result, the merit of any unknown parent is equal to zero. In reality, unknown parents are selected animals resulting from the nonrandom mating of selected parents. Therefore, depending on the generation they belong to, their selection path, and their genetic background, they can be assigned to different groups (PPG) with genetic expectation possibly different from zero. The definition of PPG is the researcher's choice, and it is population dependent. In terms of IGE for dairy cattle, it is defined based on country of origin, year of birth, and 4 pathways of selection (sires of sons, sires of daughters, dams of sons, and dams of daughters). Breed composition is also a possible component of PPG (Schaeffer, 1985). Whereas breed composition is an important factor in PPG for multibreed evaluations, in MACE the bull's country of origin is similarly important in PPG for this multiple country model, where evaluated animals are mostly purebred.

Phantom parent groups can be considered as either fixed or random effect in the model. Often, treating them as fixed effect has shown estimability problems (Schaeffer, 2018). This is mainly due to collinearity problems among PPG or between PPG and other fixed effects in the model. Therefore, treating them as random is recommended. Treating PPG as random, the PPG solutions sum to zero, and the solutions for bulls are presented relative to the same genetic base within each country (Mrode, 2005). Initially, PPG were considered as fixed effects in MACE, but by the year 2000, they were treated as random effects.

\section{Validation of NGE}

An assumption for any IGE method is the unbiasedness of NGE. An NGE is expected to remove as much bias as possible from the evaluations by correcting for 
systematic environmental effects. The NGE model is supposed to be able to make unbiased predictions of bulls' breeding values, free from all fixed effects known to be influencing the trait in that country (Schaeffer, 1985). Pedigree errors or incompleteness (Banos et al., 2001) and inaccurate parameters are other sources of bias, which also reduce accuracy of the evaluations. For accurate and unbiased results, the data upon which selection decisions have been made should be present in the data (Henderson, 1975; Kennedy et al., 1988).

The unbiasedness of IGE is tied to the unbiasedness of NGE (see, for example, Patry et al., 2013). Consequently, a common practice performed by Interbull is the validation of NGE, which is performed when a population is joining IGE for a trait, when the NGE is modified, or when an existing validation result expires (2 yr).

Interbull conducts 4 validation tests for NGE data entering MACE. The first 3 validation methods were developed by Boichard et al. (1995), and the fourth by Tyrisevä et al. (2018). In summary, the validation methods are as follows:

1. Method 1. Comparison of evaluations from data of all lactations with those of first lactations only (comparing genetic trends using first-lactation records versus all lactations to evaluate the influence of data from different daughter age groups on the genetic trend).

2. Method 2. Within-bull variation of daughter yield deviation (estimating year effect-i.e., nongenetic time trend - on bulls' DYD over years of data in the NGE).

3. Method 3. Analysis of trend in bull evaluations over time (investigating the randomness of variation in successive bull proofs).

4. Method 4. Validation of the consistency of Mendelian Sampling variance (testing the homogeneity of genetic variance and the time trend of the Mendelian Sampling variance).

\section{POST-MACE METHODS}

\section{Multiple Trait per Country MACE}

Whereas NGE usually analyze multiple traits or a trait across multiple lactations, due to the limitations of MACE, those could not be analyzed together at the international level because only one trait per country is included in a MACE analysis. If the traits had been analyzed separately at the national level, those traits cannot benefit from other within- and across-country correlated traits (i.e., only one correlated trait per country). However, if the traits had been analyzed si- multaneously at the national level, the within-country correlated trait contributions are retained in the DRP of the chosen trait to be included in MACE, but no IGE proof is obtained for the other within-country traits. Similarly, in MACE the trait would benefit from only one rather than multiple traits per country, and IGE proof is produced for one rather than multiple traits in another country. This argument can also be used for biological traits that are analyzed in multiple lactations; for instance, nationally produced proofs for separate lactations cannot be included in MACE. Consequently, within-country covariance structure is not used at the IGE level.

To remedy this, Schaeffer (2001) developed a method called multiple trait per country MACE (MT-MACE), which allowed the inclusion of multiple traits per country in IGE (i.e., nonzero residual covariances between traits from the same country). Similar to MACE (Eq. [4]), trait $j$ within country $i$ is modeled as follows:

$$
\mathbf{y}_{i j}=c_{i j} \mathbf{1}+\mathbf{Q g}_{i j}+\mathbf{s}_{i j}+\mathbf{e}_{i j}
$$

Aside from the residual variance-covariance structure and the observations (within-country traits, if more than one, should go through a multi-trait deregression procedure), the preceding equation is similar to the way that each trait is modeled in MACE (Eq. [4]). In matrix notation, MT-MACE equations for country $i$ are written as follows:

$$
\begin{aligned}
& {\left[\begin{array}{cc}
\mathbf{1}^{\prime} \mathbf{D}_{i}^{-1} \mathbf{1} & \mathbf{1}^{\prime} \mathbf{D}_{i}^{-1} \\
\mathbf{D}_{i}^{-1} \mathbf{1} & \mathbf{D}_{i}^{-1}+\mathbf{A}_{i}^{s s} \otimes \mathbf{G}^{i i} \\
\mathbf{0} & \mathbf{A}_{i}^{p s} \otimes \mathbf{G}^{i i} \\
\mathbf{0} & \mathbf{A}_{i}^{g s} \otimes \mathbf{G}^{i i}
\end{array}\right.} \\
& =\left[\begin{array}{c}
\mathbf{1}^{\prime} \mathbf{D}_{i}^{-1} \mathbf{y}_{i} \\
\mathbf{D}_{i}^{-1} \mathbf{y}_{i} \\
\mathbf{0} \\
\mathbf{0}
\end{array}\right],
\end{aligned}
$$$$
\left.\begin{array}{cc}
\mathbf{0} & \mathbf{0} \\
\mathbf{A}_{i}^{s p} \otimes \mathbf{G}^{i i} & \mathbf{A}_{i}^{s g} \otimes \mathbf{G}^{i i} \\
\mathbf{A}_{i}^{p p} \otimes \mathbf{G}^{i i} & \mathbf{A}_{i}^{p g} \otimes \mathbf{G}^{i i} \\
\mathbf{A}_{i}^{g p} \otimes \mathbf{G}^{i i} & \mathbf{A}_{i}^{g g} \otimes \mathbf{G}^{i i}
\end{array}\right]\left[\begin{array}{c}
\hat{c}_{i} \\
\hat{\mathbf{s}}_{i} \\
\hat{\mathbf{p}}_{i} \\
\hat{\mathbf{g}}_{i}
\end{array}\right]
$$

where $\mathbf{G}^{i i}$ is the block of $\mathbf{G}^{-1}$ for country $i$. Matrix $\mathbf{D}_{i}^{-1}$ should account for the nonzero within-country withinsire residual covariances. It is a block diagonal matrix with $\mathbf{D}_{i k}^{-1}$ on the diagonal for bull $k$. Daughters of bulls recorded for multiple traits are the major source of residual covariances. However, daughters might have been measured for a different combination of traits. In an example of a 3-lactation trait within a country, Schaeffer (2001) showed how $\mathbf{D}_{i k}^{-1}$ is calculated: 


$$
\mathbf{D}_{i k}^{-1}=n_{1,0,0} \mathbf{R}_{1,0,0}^{-}+n_{1,1,0} \mathbf{R}_{1,1,0}^{-}+n_{1,1,1} \mathbf{R}_{1,1,1}^{-},
$$

where $n_{1,0,0}, n_{1,1,0}$, and $n_{1,1,1}$ are the numbers of recorded daughters for the first lactation only, for the first 2 lactations only, and for all the 3 lactations, respectively, and

$$
\begin{aligned}
& \mathbf{R}_{1,0,0}^{-}=\left[\begin{array}{ccc}
r_{11}^{-1} & 0 & 0 \\
0 & 0 & 0 \\
0 & 0 & 0
\end{array}\right]
\end{aligned}
$$

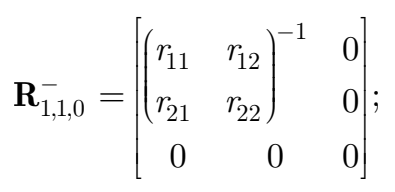

$$
\begin{aligned}
& \mathbf{R}_{1,1,1}^{-}=\mathbf{R}_{i}^{-1}=\left[\begin{array}{ccc}
r_{11} & r_{12} & r_{13} \\
r_{21} & r_{22} & r_{23} \\
r_{31} & r_{32} & r_{33}
\end{array}\right]^{-1},
\end{aligned}
$$

where $\mathbf{R}_{i}$ is the residual variance-covariance matrix among the lactations within country $i$. For the analysis of $t$ biologically distinct traits rather than $l$ lactations of a trait, the number of combinations changes from $l$ to $2^{t}-1$. For example, in the analysis of milk $(m)$, fat $(f)$, and protein $(p)$ yields (Nilforooshan, 2011),

$$
\mathbf{D}_{i k}^{-1}=\sum_{m=0}^{1} \sum_{f=0}^{1} \sum_{p=0}^{1} n_{m, f, p} \mathbf{R}_{m, f, p}^{-},
$$

where $\mathbf{R}^{-}$is the generalized inverse pertaining to the nonzero elements specific to each trait, and consequently $\mathbf{R}_{0,0,0}^{-}=0$. As a result of MT-MACE, all bulls receive evaluations for all the traits in all the countries, with the possibility of reranking across countries and across traits within a country (Nilforooshan, 2011).

Matrix $\mathbf{D}_{i}^{-1}$ (Eq. [9]) is used for both within-country multi-trait deregression (as explained by Schaeffer, 2001) of national proofs and MT-MACE. Sullivan and Wilton (2001) introduced another interpretation of MT-MACE. Whereas the method of Schaeffer (2001) was based on within-country within-sire residual weighting blocks, the method of Sullivan and Wilton (2001) was based on within-country within-sire withintrait residual weighting factors (i.e., diagonal $\mathbf{D}^{-1}$ ). The method of Sullivan and Wilton (2001) was practically more appealing, as after the derivation of the weighting factors (called effectively independent EDC by Sullivan, 2020), MT-MACE can be performed using the ordinary MACE machinery. In this interpretation of
MT-MACE, within-country traits are included in $\mathrm{MACE}$ as if they were residually independent traits. It is also difficult for the NGE centers to provide Interbull with residual weighting blocks (Schaeffer, 2001). Another advantage of the Sullivan and Wilton (2001) interpretation of MT-MACE is that it is based on EDC, rather than the number of daughters. The different options for applying MT-MACE were described and compared in detail by Sullivan et al. (2005).

Calculating EDC for MACE is performed at the national level for each trait separately. In the MT-MACE implementation of Sullivan and Wilton (2001), different EDC factors are calculated and used in the deregression procedure and MACE. The objective of the method of Sullivan and Wilton (2001) is to calculate multi-trait (residually) independent EDC that allow the inclusion of within-country traits in MACE as the equivalent of traits from different countries. This requires deriving weighting factors for a multiple-trait model considering zero residual correlations and nonzero genetic correlations among the traits (Sullivan and Wilton, 2001). In other words, the off-diagonal weights in the residual block are imposed on the diagonal values so that the resulting diagonal EDC matrices yield prediction error variances equivalent to those diagonal EDC matrices in single trait per country MACE (Sullivan, 2020).

The method of Sullivan and Wilton (2001) for the derivation of multi-trait independent EDC factors is presented in Appendix 2. Despite many studies performed on MT-MACE (Schaeffer, 2001; Sullivan and Wilton, 2001; Sullivan et al., 2005; Mark and Sullivan, 2006; Nilforooshan et al., 2010a, 2014), it has never been officially adopted for IGE.

\section{Genomic MACE}

By 2010, many Interbull member countries had already implemented or were in the process of implementing genomic BLUP (GBLUP; VanRaden, 2008) to enhance their NGE. There was an increasing demand by the industry for having genomically enhanced IGE, mainly to facilitate worldwide marketing of genetic materials from young genotyped bulls without progeny, or without enough progeny to receive an official proof. VanRaden and Sullivan (2010) introduced genomic MACE (GMACE) methodology to accommodate national genomic evaluations (genomic EBV, GEBV) into IGE. Interbull Centre soon adopted this method for pilot studies, and in August 2014 the first official GMACE results were released for the Holstein breed and all the 7 trait groups (Tables 1 and 2) to the participating countries.

Genomic information can be transferred among countries by combining their national GEBV (VanRaden 
and Sullivan, 2010), genotypes, or national genomic equations (Goddard et al., 2018). In GMACE, genomic information is transferred via $\mathrm{A}^{-1}$ from deregressed national GEBV with reciprocals of genomically enhanced EDC used as residual weights. Compared with MACE, GMACE uses genomically enhanced EDC instead of EDC, and national GEBV instead of national EBV. Residuals between countries are no longer independent if countries use foreign data (e.g., MACE proofs) regressing on genotypes, or for predicting marker effects in their genomic NGE (VanRaden and Sullivan, 2010). Similar to MT-MACE, GMACE deals with nonzero residual covariances. Theoretically, it is possible considering more than one trait per country in GMACE, but in practice one trait per country is included so far. Therefore, nonzero residual covariances are limited to within sire across country. The same methodology that made possible the interpretation of MT-MACE using the MACE procedure (Sullivan and Wilton, 2001) also made possible the implementation of GMACE using the MACE procedure.

Another difference between MACE and GMACE is in the approximation of reliabilities. The MACE reliabilities are approximated by the method of Harris and Johnson (1998), and a modified version is used for MT-MACE (Mark and Sullivan, 2006). Neither of these methods account for nonzero residual correlations between countries (Sullivan and VanRaden, 2009). Sullivan (2013) made improvements to the reliability approximation for GMACE.

Additional effective daughter equivalents from genomic information $\left(\mathrm{EDC}_{g e n}\right)$ are added to the effective daughter equivalents without genomic information (EDC), applied to the diagonal elements of $\mathbf{D}^{-1}$. The residual covariances created by daughter equivalents from foreign genomic information is a function of the number of common genotyped bulls between the 2 countries, the genetic correlation between the 2 countries, and $\mathrm{EDC}_{g e n} /\left(\mathrm{EDC}_{d a u}+\mathrm{EDC}_{g e n}\right)$ in each country, where $\mathrm{EDC}=\mathrm{EDC}_{p a}+\mathrm{EDC}_{d a u}, \mathrm{EDC}_{p a}$ is the daughter equivalent contribution from parent average, and $\mathrm{EDC}_{d a u}$ is the effective daughter contribution from daughters themselves (VanRaden and Sullivan, 2010).

Like MACE, data used in GMACE go through a validation procedure. Mäntysaari et al. (2010) proposed a validation procedure for national genomic evaluations in GMACE. This procedure is well-explained and tested by Nilforooshan et al. (2010b).

\section{SNP-MACE}

Genomic predictions can be made in various ways, including GBLUP, BLUP at the SNP level (SNPBLUP), single-step GBLUP (ss-GBLUP), or single- step marker effect model (ssMEM; Fernando et al., 2014). Koivula et al. (2012) made comparisons among GBLUP, ss-GBLUP, and SNP-BLUP. Unlike the first 2 methods, which estimate genomic predictions at the animal level using genomic relationship matrices, the latter estimates genomic predictions at the SNP level (i.e., BLUP estimates of marker effects, assuming a normal distribution and equal variance for all markers; Koivula et al., 2012). Then, animals' direct genomic values can be calculated by summing up allele substitution effects (i.e., SNP effects obtained from SNP-BLUP) over genotype across all markers. Liu et al. (2016) extended SNP-BLUP to include residual polygenic effects (additive genetic variance not explained by the markers due to causal variants in other parts of the genome and incomplete linkage disequilibrium between the SNP markers and the causal variants). Fitting residual polygenic effects in genomic evaluations is important to reduce the inflation of genomic predictions (Liu et al., 2016; Nilforooshan, 2020).

The SNP-MACE method (Goddard et al., 2018) has been proposed as a possible method of IGE for the future. Interbull has formed a working group for the methodological development of SNP-MACE. According to Goddard et al. (2018), the main distinction between SNP-MACE and a multiple-trait SNP-BLUP is that SNP-MACE is a meta-analysis combining SNP solutions from different countries. For $t$ countries, the SNP-MACE model is as follows:

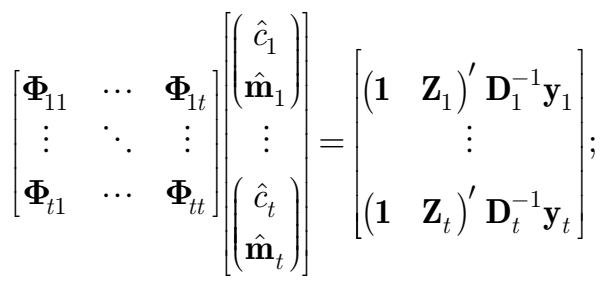

$$
\begin{aligned}
& \boldsymbol{\Phi}_{i i}=\left[\begin{array}{cc}
\mathbf{1}^{\prime} \mathbf{D}_{i}^{-1} \mathbf{1} & \mathbf{1}^{\prime} \mathbf{D}_{i}^{-1} \mathbf{Z}_{i} \\
\mathbf{Z}_{i}^{\prime} \mathbf{D}_{i}^{-1} \mathbf{1} & \mathbf{Z}_{i}^{\prime} \mathbf{D}_{i}^{-1} \mathbf{Z}_{i}+\mathbf{G}^{i i}
\end{array}\right] ; \\
& \boldsymbol{\Phi}_{i j \mid i \neq j}=\left[\begin{array}{cc}
0 & \mathbf{0} \\
\mathbf{0} & \boldsymbol{\Psi}_{i j}+\mathbf{G}^{i j}
\end{array}\right] ; \\
& \mathbf{\Psi}_{i j}=\left(\mathbf{Z}_{i}^{\prime} \mathbf{D}_{i}^{-0.5}\right)\left(\mathbf{D}_{j}^{-0.5} \mathbf{Z}_{j}\right) .
\end{aligned}
$$

Equation [15] is for the situation where the countries share data and use deregressed MACE proofs containing common daughter information in their genomic NGE. If the 2 countries use only national phenotypes to estimate SNP effects, then $\boldsymbol{\Psi}_{i j}=\mathbf{0}$. Matrix $\boldsymbol{\Phi}_{i i}$ is the left-hand-side of the national SNP model from country 
$i$, and $\boldsymbol{\Psi}_{i j}$ is the residual covariance co-incidence matrix between SNPs in countries $i$ and $j$. Vector $\mathbf{y}_{i}$ is a vector of observations (DRP) of reference animals, $\mathbf{Z}_{i}$ is the design matrix for genotypes in country $i$, coded as $1-$ $2 p_{i j}$ for the heterozygotes, $2-2 p_{i j}$, and $-2 p_{i j}$ for the 2 types of homozygotes (genotypes must be coded in the same way in different countries), $p_{i j}$ is the allele frequency of marker $j$ in country $i$, $\mathbf{D}_{i}=\operatorname{Var}\left(\mathbf{e}_{i}\right)=\operatorname{diag}\left(\sigma_{e_{i}}^{2} / n_{i k}\right), \sigma_{e_{i}}^{2}$ is the residual variance in country $i, n_{i k}$ is the daughter contribution of animal $k$ in country $i$ based on the reliabilities of the bull and its parents, $\hat{c}_{i}$ is the country $(i)$ mean estimate expressed on the scale of direct genomic values (rather than GEBV), $\hat{\mathbf{m}}_{i}$ is the vector of SNP effect solutions on the scale of country $i$, and

$$
\left[\begin{array}{ccc}
\mathbf{G}^{11} & \cdots & \mathbf{G}^{1 t} \\
\vdots & \ddots & \vdots \\
\mathbf{G}^{t 1} & \cdots & \mathbf{G}^{t t}
\end{array}\right]=\mathbf{G}^{-1}
$$

Relying on the assumption that the same set of $m$ SNP markers are used in $t$ countries,

$$
\begin{gathered}
\operatorname{Var}(\mathbf{g})=\mathbf{G}=\left[\begin{array}{ccc}
\sigma_{11} \theta_{11} & \cdots & \sigma_{1 t} \theta_{1 t} \\
\vdots & \ddots & \vdots \\
\sigma_{t 1} \theta_{t 1} & \cdots & \sigma_{t t} \theta_{t t}
\end{array}\right] \otimes \mathbf{I}_{m} \\
1 / \theta_{i i}=\sum_{j=1}^{m} 2 p_{i j}\left(1-p_{i j}\right) \\
\theta_{i k}=\sqrt{\theta_{i i} \theta_{k k}}
\end{gathered}
$$

where $\mathbf{I}_{m}$ is an identity matrix with the size of $m$ number of SNPs, and $\theta$ values are scaling factors taking into account allele frequencies across genotypes. The model assumes that all SNP effects in different countries are genetically correlated with the same genetic correlations as the correlations between predicted genetic merits. We think that $\mathbf{D}_{i}^{-0.5} \mathbf{D}_{j}^{-0.5}$ in Eq. [15] may need to be multiplied by $r^{i j}$, which is the element in the inverse of the residual correlation matrix corresponding to countries $i$ and $j$, as $\mathbf{D}_{i}^{-0.5} \mathbf{D}_{j}^{-0.5}$ implies a residual correlation of 1 between countries $i$ and $j$.

The SNP-MACE method involves exchanging national genomic equations $\left[\boldsymbol{\Phi}_{i i}\right.$ and $\left(\begin{array}{ll}\mathbf{1} & \mathbf{Z}_{i}\end{array}\right)^{\prime} \mathbf{D}_{i}^{-1} \mathbf{y}_{i}$ for country $i$ ] between countries and Interbull. Information between countries is exchanged for $\hat{\mathbf{m}}_{i}$ solutions via $\boldsymbol{\Psi}_{i j}$ $+\mathbf{G}^{i j}$ (Eq. [14]). Combining data over countries is expected to increase the accuracy of SNP effects and therefore GEBV. Moreover, the accuracy of GEBV de- pends on the proportion of genetic variance explained by the SNP markers.

Usually, countries use different sets of SNPs in their genomic evaluations, using different genotyping chips or different sets of SNPs from the same chip. The latter may happen after the quality control procedure. Limiting the number of equations to SNPs in common between all countries, a lot of information and genetic variation could be lost. One recommendation would be to use SNPs used in more than one country. As a result, $\boldsymbol{\Phi}_{i i}$ and $\boldsymbol{\Phi}_{j j}$ are square matrices with different sizes and $\boldsymbol{\Phi}_{i j}$ is a rectangular matrix. A square block of $\boldsymbol{\Phi}_{i j}$ contains SNPs in common between countries $i$ and $j$. With equations for country $i$ appearing before equations for country $j, \boldsymbol{\Phi}_{i j}$ would have rows of zeros for SNPs in country $i$ but not in country $j$, and columns of zeros for SNPs in country $j$ but not in country $i$. The structure of $\mathbf{G}^{-1}$ would need to change as well, and $\mathbf{G}_{i j}$ would become a rectangular matrix. Generally, the benefit from SNP-MACE is expandable to genotyped animals from the participating countries that were not present in the analysis. This is achieved using conversion equations. However, if the set of SNPs used in different countries are different, sharing genotypes or re-genotyping the same animal in the other country is needed to calculate GEBV of the animal on the base and scale of the other country. Otherwise, a nonoptimal solution would be to calculate GEBV of the animal on the base and scale of the other country, based on the SNPs that the 2 countries have in common. The SNP-MACE method potentially benefits GMACE. The SNP-MACE solutions converted to GEBV can be used as input to GMACE if the corresponding input is missing in GMACE (Sullivan, 2020).

\section{GENOMIC ERA AND GENOMIC PRESELECTION}

Commercial production of genotyping arrays with a massive number of SNPs paved the way for implementing the genomic methods suggested by Meuwissen et al. (2001). However, it was not clear how the new SNP data would be integrated into animal breeding programs, and how the structure of such programs would be affected by such implementations. Schaeffer's (2006) article on applying genome-wide selection in dairy cattle stirred interest among dairy cattle researchers. The contributions of Van Tassell et al. (2008) toward the design of a bovine chip, and of VanRaden (2008) toward the design of the statistical methods to analyze SNP data, were crucial in making the first large-scale genomic evaluation of dairy cattle, at the USDA, a reality. The Interbull community was very quick to form a genomic task force so that possible scenarios for genomic IGE could be examined. 
The preliminary report of the Interbull task force (Banos et al., 2009) envisaged many scenarios, the first of which was for the Interbull Centre to access national predicted genetic merits and genotype of bulls. If that were the case, then the Interbull Centre would pool genotypes to form a common reference population and use the MACE proofs to start a pseudo-national genomic evaluation on behalf of the participating countries. The size and the structure of the reference population size greatly influences the accuracy of genomic estimated breeding values (Hayes et al., 2009). The reference population size for many national dairy cattle breeds is small. Consequently, pooling national reference populations into an international reference population is greatly beneficial, especially for small breeds. The InterGenomics project is about pooling genotypes of bulls with MACE proof from multiple countries for building a common reference population and performing a multinational genomic evaluation based on the GBLUP methodology (VanRaden, 2008). At first, several European and the US Brown Swiss populations joined InterGenomics (Jorjani et al., 2010), but later this project was also adopted by several Holstein populations (Jorjani et al., 2017).

Availability of genomic evaluations for young bulls makes it possible to distinguish among full-sibs, for different Mendelian Sampling terms. Consequently, nextgeneration cows will not be from a random sample of sons. This will create bias in national and international genetic evaluation (Patry et al., 2013). From a theoretical point of view, the implementation of genomics in dairy cattle may aggravate violations of underlying assumptions of the BLUP animal model (Schaeffer, 2018). These violations can potentially lead to bias in national and, consequently, international genetic evaluations. Sullivan et al. (2019) have proposed using newer methods to minimize these potential biases in both NGE and IGE, by improving the model assumptions and accounting for genomic preselection effects.

The use of genomic selection in the national evaluations has also changed the structure of dairy cattle populations, in the sense that the turnover of young bulls has become much faster. This has 2 consequences. The first is that the semen of young bulls is used during a short period of time, and consequently their offspring are born during a short period of time. The second is that the generation path from sire to son has become so short that some bulls become paternal grandsires before they have enough daughters with records to receive official proofs to be considered in IGE. These 2 consequences have an indirect effect on IGE, because it becomes increasingly challenging to find enough bulls to perform validation tests.

\section{FUTURE OF MACE AND IGE}

To date, IGE has been officially practiced for 6 dairy breed groups (Holstein, Brown Swiss, Guernsey, Jersey, Red Dairy Cattle, and Simmental) and 5 beef breed groups (Charolais, Limousine, Angus, Hereford, and Simmental). Similar attempts have been initiated for horses (Bruns et al., 2004; Thorén Hellsten et al., 2008, 2009; Ruhlmann et al., 2009) under the project Interstallion, also using MACE. For IGE of beef, MACE was not a good candidate because (1) some countries did not have an NGE for beef, and thus, MACE was out of the question for them; (2) daughter groups are considerably smaller for beef than for dairy breeds, so that there is less benefit in using MACE; and (3) unlike dairy traits, males have phenotypes in beef traits.

Generally, a multi-trait animal model with national data from multiple countries combined is used for the IGE of beef. Phenotypic data from each country is considered as a different trait, possibly with a different model. Interbeef (a working group of the International Committee for Animal Recording) is leading the IGE of 5 beef breeds, 8 populations in 10 European countries, and 2 trait groups (weaning weight and calving, the latter including calving ease and birth weight; Bonifazi et al., 2020).

With concerns over the unbiasedness of national BLUP in the presence of genomic preselection (Patry et al., 2013; Schaeffer, 2018), there are concerns about the unbiasedness of MACE and its life expectancy. Genomic selection has been extensively carried out for the Holstein breed in many countries. Therefore, we can say that at least for the Holstein breed, the national bias may render the current MACE application inadequate. However, MACE has continued to be used, and may do so for some time in the future, because the amount of bias has not yet been high enough to make MACE completely obsolete. The fact that different NGE still manage to pass the Interbull validation tests indicates that bias is still low. Additionally, no strong candidate and practically feasible method has been proposed to replace MACE. Debate exists about the amount of genomic preselection bias in practice, as it may be reduced by accumulating daughters over time (Sullivan, 2020). Therefore, this bias may mainly target young bulls. Although the overall bias may show reduction over time, genetic trends are biased in different subsets of population in different ways (Masuda et al., 2018).

Many studies have assessed the bias of genomic preselection in simulated data (e.g., Jibrila et al., 2020). However, few studies have examined within- and acrosscountry genomic preselection bias on real data (e.g., Patry et al., 2013; Wang et al., 2020). Depending on 
the extent of genomic preselection and its accuracy, its bias can differ from one population to another. Validation of NGE with emphasis on genomic preselection and empirical estimates of its bias is necessary. Methods are already in place for this (Mäntysaari et al., 2010; Nilforooshan et al., 2010b; Tyrisevä et al., 2018); however, further research is necessary. The extent of genomic preselection in breeds other than Holstein-Friesian does not seem to be at a level to rule out MACE for those breeds. Therefore, MACE may still be used for those breeds as well as for other species.

In the future, MACE may be used for purposes other than IGE. Generally, wherever residually independent variables are to be analyzed together, MACE is a possible candidate. For residually correlated traits, MTMACE is a candidate to combine EBV of traits from separate analyses. Due to computational limitations, countries may not be able to analyze raw phenotypes on all traits in a multiple-trait model. Therefore, they analyze traits in single-trait models or groups of multiple-trait models, and then combine the solutions using selection index theory (Weigel, 1996; VanRaden, 2001). The MACE method can achieve more accurate results than a selection index due to a properly weighted system of genetic and residual (variances) information. The first step is obtaining residually independent DRP from EBV. Then, those DRP can be analyzed by the MACE machinery. Assuming national traits are analyzed in single-trait models, the traits can be deregressed using a single-trait deregression procedure and single-trait EDC factors, but because MACE does not enforce nonzero residual correlations, the residual correlations between traits should be enforced via multi-trait EDC factors in MACE (Sullivan and Wilton, 2001). The work of Sullivan and Wilton (2001) increased MACE's life expectancy in a situation where residually correlated traits (e.g., traits from the same country) are to be used in IGE.

Confusion arises when some of the traits had been previously analyzed together in a multiple-trait model. For those traits, the residual contribution of traits to each other are already accounted for. We suggest 2 approaches: (1) multi-trait deregression of the EBV (of the traits analyzed together) using multi-trait EDC factors to remove the residual contributions of the traits on each other, and then reinforcing those contributions in the re-regression step; (2) assuming the same pedigree used for all the traits, ignoring residual correlations in the deregression procedure (preserving the residual contributions), and then skipping reinforcing those contributions in the re-regression step. The re-regression step can be either MACE using multi-trait EDC factors (Sullivan and Wilton, 2001) or MT-MACE (Schaeffer,
2001). Considering an example of an NGE with 4 traits, where traits 1 and 2 and traits 3 and 4 are analyzed in 2 multiple-trait models, the first approach would be deregressing the 2 groups of traits separately, taking into account the residual correlations between traits 1 and 2 and between traits 3 and 4 , and then considering all the (6) residual correlations in multi-trait EDC factors used in MACE or multi-trait EDC blocks used in MTMACE. The second approach would be de-regressing EBV for each trait independently, ignoring residual correlations, and then reinforcing residual correlations except those between traits 1 and 2 and between traits 3 and 4 , in multi-trait EDC factors used in MACE or multi-trait EDC blocks used in MT-MACE.

Assuming the same pedigree is used for all the traits in an NGE, all animals receive EBV for all the traits. That provides great opportunities for data transformation. One possibility is to transform EBV to residually independent variables before deregression. Such transformation for animal $i$ involves $\mathbf{y}_{i}^{*}=\boldsymbol{\Gamma} \mathbf{y}_{i}$, where $\mathbf{y}$ represents EBV from the NGE model, $\boldsymbol{\Gamma R}_{i} \boldsymbol{\Gamma}^{\prime}=\operatorname{diag}\left(\mathbf{k}_{i}\right)$. Matrix $\boldsymbol{\Gamma}$ makes y elements residually independent, $\mathbf{R}_{i}$ is the residual variance-covariance matrix for animal $i$, and vector $\mathbf{k}$ is different from one animal to another due to the different accuracy of each trait's proof. Decomposing the residual correlation matrix among the traits to its eigenvalues $\left(\mathbf{B}^{2}\right)$ and eigenvectors $(\mathbf{U}), \mathbf{P}=$ UBU' $^{\prime}$. Then $\boldsymbol{\Gamma}=\mathbf{L}^{\prime} \mathbf{P}$, where $\mathbf{L}$ is the matrix of eigenvectors for $\mathbf{P P}^{\prime}$. Then, MACE solutions should be back-transformed to the original scale $\left(\boldsymbol{\Gamma}^{-1} \hat{\mathbf{u}}_{i}^{*}=\hat{\mathbf{u}}_{i}\right.$, where $\hat{\mathbf{u}}_{i}^{*}=\mathbf{Q} \hat{\mathbf{g}}_{i}^{*}+\hat{\mathbf{s}}_{i}^{*}$ is the vector of MACE solutions for animal $i$ on the transformed scale).

The SNP-MACE method is a strong candidate as an additional service along with MACE and GMACE. The 2 major advantages of SNP-MACE are (1) the number of SNPs does not grow as fast as the number of genotyped animals, so that fewer equations need to be solved; and (2) it directly benefits genotyped animals included in the analysis, and indirectly benefits other genotyped animals in the participating countries via SNP conversion equations. A disadvantage of SNP$\mathrm{MACE}$ is that it has nothing to offer for nongenotyped animals. A future development in SNP-MACE might incorporate residual polygenic effects, similar to the equivalent SNP-BLUP with residual polygenic effects (Liu et al., 2016). It is not guaranteed that SNP-MACE would be free from genomic preselection bias, as preselection of animals based on their GEBV constitutes preselection on different SNP alleles, and changing allele frequencies. National and international genomic evaluations (both GMACE and SNP-MACE) become biased due to the use of DRP in genomic NGE, to lesser degrees compared with evaluations without genomic 
information but perhaps similarly for GMACE relative to SNP-MACE (Sullivan, 2020).

Fulfilling the underlying assumptions of Eq. [12-15] poses some practical difficulties for SNP-MACE. Countries need to use only the bull proofs that are based on domestic daughters. If the exchange of female genotypes across countries becomes more widespread, special attention should be given to bull dams so that any residual correlations can be avoided. The restriction on the use of foreign data may force countries to have 2 evaluations, one before a genomic IGE and one after. Further, given the differences in breeding objectives in different countries, it is not clear whether all countries can agree on the same set of SNPs to be used. It may also be that the minor allele frequency of some alleles in some countries is below the threshold of data edits. Furthermore, if the national genomic model assigns zero variance to any SNP, the effects of such SNPs from an international genomic model will be of limited use for that country and lead to missing variance.

It is difficult to know where the future of animal breeding and IGE lies. However, given that a new era of animal breeding has begun, using genomics, it is difficult to imagine the future of IGE without genomics. The number of genotyped animals is increasing rapidly. With the number of genotyped animals exceeding the number of SNPs, singularity problems would arise in some animal models that involve a genomic relationship matrix with the size equal to the number of genotyped animals, and estimating SNP effects is more practical and beneficial than estimating individuals' genetic merits. Furthermore, not every individual is being genotyped, and an ideal model would include information on both genotyped and non-genotyped animals. The ss-GBLUP method for simultaneous genetic evaluation of genotyped and non-genotyped animals (Aguilar et al., 2010; Christensen and Lund, 2010) has received a great deal of attention from animal breeders around the world. It involves replacing $\mathbf{A}^{-1}$ in BLUP with $\mathbf{H}^{-1}$, which involves both pedigree and genomic information. Due to the inclusion of both genotyped and non-genotyped animals in the model, bias from genomic preselection is reduced considerably. Missing genotype and phenotype information for certain animals may introduce slight bias to ss-GBLUP (Jibrila et al., 2020, 2021). Simultaneous evaluation of genotyped and nongenotyped animals is also appealing for IGE. However, fitting MACE with an $\mathbf{H}^{-1}$ matrix does not seem to be a feasible option for numerous reasons: (1) difficulty and lack of willingness in sharing genotypes globally by NGE centers; (2) computational challenges of calculating international $\mathbf{G}^{-1}$ and $\mathbf{A}_{22}^{-1}$ matrices by the Interbull Centre, where $\mathbf{G}$ is the genomic relationship matrix between animals, and $\mathbf{A}_{22}$ is the block of $\mathbf{A}$ corresponding to genotyped animals; (3) different genotypes or different sets of SNP markers from the same chip are used by the NGE, and an international imputation to a defined or desired set of SNP markers would be another challenge for the Interbull Centre; (4) conflicting genotypes among if not within countries; (5) different countries may prefer different weights of blending $\mathbf{G}$ and $\mathbf{A}_{22}$; and more. Despite single-step methods becoming more popular, their application is generally limited to NGE (Sullivan, 2020). Nevertheless, a single-step IGE, if technically and practically possible, should be pursued. More and more countries are converting their NGE system to ss-GBLUP. Although sire proofs from those evaluations can be used in GMACE, they cannot be used as input to MACE, because genotypes are shared multinationally. Similarly, for the 2-step genomic NGE, MACE proofs are often used as input. Therefore, countries need to keep their BLUP evaluations in place to be able to submit data to MACE, in its current form (Sullivan, 2020).

One of the limitations of MACE, rooted in the use of DRP, is the addition of artificial interaction to $G \times$ E. This limitation is not specific to MACE but applies in other IGE methods too. This artificial interaction between sire proofs comes from different data editing procedures and genetic evaluation models, leading to genetic correlations less than unity even if no biological $\mathrm{G} \times \mathrm{E}$ exists (Mrode, 2005). By contrast, $\mathrm{G} \times \mathrm{E}$ within large countries might be greater than $\mathrm{G} \times \mathrm{E}$ across small countries, or countries with similar climates and production systems (Rekaya et al., 2001). A few studies have suggested borderless evaluations based on multiple-trait herd cluster models, grouping herds across countries based on similar production environments (Lohuis and Dekkers, 1998; Weigel and Rekaya, 2000).

When it comes to developing and choosing a method for future IGE, as always, practical considerations and the type of data possible to share with Interbull are crucial factors (Sullivan, 2020).

\section{SUMMARY}

Genetic exchange across countries will continue. In the past, the major flow was from the North American countries to other countries. In the future, the exchange flow is expected to be multidirectional. The global reduction in the effective population sizes is something to be addressed. The MACE method has been the most long-lasting and reliable method of IGE, and subsequent methods such as MT-MACE and GMACE are built upon its structure. In the new era of genomics, genomic preselection has caused bias in national BLUP 
and, by extension, in MACE evaluations. This bias also influences other forms of NGE, directly and indirectly, if DRP from BLUP are used as input information. The scale of genomic preselection is less severe in breeds other than Holstein. Therefore, MACE may continue to be used for the non-Holstein breeds, or even for other species or for purposes other than IGE. By contrast, countries may choose to continue using MACE proofs if they continue using their national BLUP evaluations, accepting that their national BLUP evaluations might be biased and that bias is introduced to MACE by the other participating countries in the analysis. Bias from different countries might be aggregative or lessening, depending on selection decisions in each country. Genomics is expected to play a major role in the future of IGE. Although SNP-MACE might be seen as a future alternative to MACE, it might not be an ultimate alternative, as it does not address non-genotyped animals. Also, the willingness of countries to share genotypes and SNP effects is presently unclear. It might not be possible now, but a future IGE may involve exchanging genotypes between countries and Interbull. The successful experience with the InterGenomics project for the Brown Swiss breed has shown that this is possible. Bias from genomic preselection remains an issue, and a method to systematically correct for it would be a breakthrough. Assumptions on random Mendelian Sampling and its normal distribution might need to change, and the expectancy of the amount of skewness that each animal contributes to the distribution might need to be approximated. Meanwhile, MACE is continuing to be used, and could remain relevant for years to come, but the beginning of its end has probably begun.

\section{ACKNOWLEDGMENTS}

The authors thank Esa Mäntysaari (LUKE, Helsinki, Finland) and Peter Sullivan (Lactanet, Sainte-Annede-Bellevue, Canada) for their feedback, which helped improve this work. The authors have not stated any conflicts of interest.

\section{REFERENCES}

Aguilar, I., I. Misztal, D. L. Johnson, A. Legarra, S. Tsuruta, and T. J. Lawlor. 2010. Hot topic: A unified approach to utilize phenotypic, full pedigree, and genomic information for genetic evaluation of Holstein final score. J. Dairy Sci. 93:743-752. https://doi.org/10 $.3168 /$ jds.2009-2730.

Bagnato, A., A. Rossoni, C. Nicoletti, J. Jakobsen, and E. Santus. 2007. Milkability and temperament MACE correlation and pilot study in dairy cattle populations. Interbull Bull. 37:95-97. https:/ /journal.interbull.org/index.php/ib/article/view/993.

Banos, G., M. Calus, V. Ducrocq, J. Dürr, H. Jorjani, Z. Liu, E. Mäntysaari, P. Sullivan, and P. VanRaden. 2009. Preliminary re- port from Interbull Task Force on the role of genomic information in genetic evaluations. Interbull Bull. 39:61-65. https://journal .interbull.org/index.php/ib/article/view/1067.

Banos, G., L. R. Schaeffer, and E. E. Burnside. 1991. Genetic relationships and linear model comparisons between United States and Canadian Ayrshire and Jersey bull populations. J. Dairy Sci 74:1060-1068. https://doi.org/10.3168/jds.S0022-0302(91)78256 -8 .

Banos, G., G. R. Wiggans, and R. L. Powell. 2001. Impact of paternity errors in cow identification on genetic evaluations and international comparisons. J. Dairy Sci. 84:2523-2529. https://doi.org/10 .3168/jds.S0022-0302(01)74703-0.

Boichard, D., B. Bonaiti, A. Barbat, and S. Mattalia. 1995. Three methods to validate the estimation of genetic trend for dairy cattle. J. Dairy Sci. 78:431-437. https://doi.org/10.3168/jds.S0022 $-0302(95) 76652-8$.

Bonifazi, R., J. Vandenplas, J. ten Napel, K. Matilainen, R. F. Veerkamp, and M. P. L. Calus. 2020. Impact of sub-setting the data of the main Limousin beef cattle population on the estimates of across-country genetic correlations. Genet. Sel. Evol. 52:32. https://doi.org/10.1186/s12711-020-00551-9.

Bruns, E., A. Ricard, and E. Koenen. 2004. Interstallion-On the way to an international genetic evaluation of sport horses. Page 326 in Proc. 55th Euro. Assoc. Anim. Prod. Wageningen Academic Publishers.

Christensen, O. F., and M. S. Lund. 2010. Genomic prediction when some animals are not genotyped. Genet. Sel. Evol. 42:2. https:// doi.org/10.1186/1297-9686-42-2.

de Jong, G. 2003. MACE-Options for improvement. Interbull Bull. 30:112-116. https://journal.interbull.org/index.php/ib/article/ view/784.

Falconer, D. S. 1952. The problem of environment and selection. Am. Nat. 86:293-298. https://doi.org/10.1086/281736.

Fernando, R. L., J. C. Dekkers, and D. J. Garrick. 2014. A class of Bayesian methods to combine large numbers of genotyped and non-genotyped animals for whole-genome analyses. Genet. Sel. Evol. 46:50. https://doi.org/10.1186/1297-9686-46-50.

Fikse, W. F., and G. Banos. 2001. Weighting factors of sire daughter information in international genetic evaluations. J. Dairy Sci 84:1759-1767. https://doi.org/10.3168/jds.S0022-0302(01)74611 -5 .

Fikse, W. F., and P. G. Sullivan. 1999. Use of national reliability figures to re-engineer effective number of records for application in international genetic evaluations. Interbull Bull. 22:44-48. https:/ /journal.interbull.org/index.php/ib/article/view/411.

Garrick, D. J., J. F. Taylor, and R. L. Fernando. 2009. Deregressing estimated breeding values and weighting information for genomic regression analyses. Genet. Sel. Evol. 41:55. https://doi.org/10 $.1186 / 1297-9686-41-55$.

Goddard, M. 1985. A method of comparing sires evaluated in different countries. Livest. Prod. Sci. 13:321-331. https://doi.org/10.1016/ 0301-6226(85)90024-7.

Goddard, M. E., A. Jighly, H. Benhajali, J. Jorjani, and Z. Liu. 2018. SNPMace - A meta-analysis to estimate SNP effects by combining results from multiple countries. Interbull Bull. 54:13-18. https:// journal.interbull.org/index.php/ib/article/view/1464.

Guo, J., H. Jorjani, and Ö. Carlborg. 2012. A genome-wide association study using international breeding-evaluation data identifies major loci affecting production traits and stature in the Brown Swiss cattle breed. BMC Genet. 13:82. https://doi.org/10.1186/ 1471-2156-13-82.

Harris, B., and D. Johnson. 1998. Approximate reliability of genetic evaluations under an animal model. J. Dairy Sci. 81:2723-2728. https://doi.org/10.3168/jds.S0022-0302(98)75829-1.

Harris, B. L., and E. S. Kolver. 2001. Review of Holsteinization on intensive pastoral dairy farming in New Zealand. J. Dairy Sci. 84:E56-E61. https://doi.org/10.3168/jds.S0022-0302(01)70197-X.

Hayes, B. J., P. J. Bowman, A. C. Chamberlain, K. Verbyla, and M. E. Goddard. 2009. Accuracy of genomic breeding values in multibreed dairy cattle populations. Genet. Sel. Evol. 41:51. https://doi .org/10.1186/1297-9686-41-51. 
Henderson, C. R. 1975. Best linear unbiased estimation and prediction under a selection model. Biometrics 31:423-447. https://doi.org/ $10.2307 / 2529430$.

Henderson, C. R. 1976. A simple method for computing the inverse of a numerator relationship matrix used in prediction of breeding values. Biometrics 32:69-83. https://doi.org/10.2307/2529339.

Huang, W., M. A. Carbone, R. F. Lyman, R. R. H. Anholt, and T. F. C. Mackay. 2020. Genotype by environment interaction for gene expression in Drosophila melanogaster. Nat. Commun. 11:5451. https://doi.org/10.1038/s41467-020-19131-y.

International Dairy Federation (IDF). 1981. IDF Recommended Procedure for International Comparison of Genetic Merit of Dairy Cattle. International Dairy Federation.

Jairath, L., J. C. M. Dekkers, L. R. Schaeffer, Z. Liu, E. B. Burnside, and B. Kolstad. 1998. Genetic evaluation for herd life in Canada. J. Dairy Sci. 81:550-562. https://doi.org/10.3168/jds.S0022 -0302(98)75607-3.

Jakobsen, J. H., and J. W. Dürr. 2012. Implementing a sire-dam pedigree structure in MACE. Interbull Bull. 45:1-6. https://journal .interbull.org/index.php/ib/article/view/1242.

Jakobsen, J. H., V. Palucci, and H. Jorjani. 2008. Feasibility of international genetic evaluation for workability traits. Interbull Bull. 38:101-106. https://journal.interbull.org/index.php/ib/article/ view/1043.

Jibrila, I., J. ten Napel, J. Vandenplas, R. F. Veerkamp, and M. P. L. Calus. 2020. Investigating the impact of preselection on subsequent single-step genomic BLUP evaluation of preselected animals. Genet. Sel. Evol. 52:42. https://doi.org/10.1186/s12711-020 $-00562-6$

Jibrila, I., J. Vandenplas, J. ten Napel, R. F. Veerkamp, and M. P. L. Calus. 2021. Avoiding preselection bias in subsequent single-step genomic BLUP evaluations of genomically preselected animals. J. Anim. Breed. Genet. 138:432-441. https://doi.org/10.1111/jbg .12533 .

Jorjani, H. 2005. Interbull pilot study for female fertility traits in Holstein populations. Interbull Bull. 33:34-46. https://journal .interbull.org/index.php/ib/article/view/871.

Jorjani, H., L. Klei, and U. Emanuelson. 2003. A simple method for weighted bending of genetic (co)variance matrices. J. Dairy Sci. 86:677-679. https://doi.org/10.3168/jds.S0022-0302(03)73646-7.

Jorjani, H., V. Palucci, and J. Sendecka. 2017. InterGenomics for Holstein populations. In 2017 Interbull Annual Meeting, August 25-28, 2017, Tallinn, Estonia. Accessed Mar. 22, 2021. https:// interbull.org/static/web/09_30_Jorjani.pdf.

Jorjani, H., J. Philipsson, and J.-C. Mocquot. 2001. Interbull guidelines for national \& international genetic evaluation systems in dairy cattle with focus on production traits. Interbull Bull. 28:127. https://journal.interbull.org/index.php/ib/issue/view/17.

Jorjani, H., B. Zumbach, J. Dürr, and E. Santus. 2010. Joint genomic evaluation of BSW populations. Interbull Bull. 41:8-13. https:// journal.interbull.org/index.php/ib/article/view/1133.

Kennedy, B. W., L. R. Schaeffer, and D. A. Sorensen. 1988. Genetic properties of animal models. J. Dairy Sci. 71:17-26. https://doi .org/10.1016/S0022-0302(88)79975-0.

Klei, L., and T. J. Lawlor. 1998. MACE for conformation traits. Interbull Bull. 17:64-69. https://journal.interbull.org/index.php/ib/ article/view/487.

Koivula, M., I. Strandén, G. Su, and E. A. Mäntysaari. 2012. Different methods to calculate genomic predictions - Comparisons of BLUP at the single nucleotide polymorphism level (SNP-BLUP), BLUP at the individual level (G-BLUP), and the one-step approach $(\mathrm{H}-$ BLUP). J. Dairy Sci. 95:4065-4073. https://doi.org/10.3168/jds 2011-4874.

Kolmodin, R., E. Strandberg, P. Madsen, J. Jensen, and H. Jorjani. 2002. Genotype by environment interaction in Nordic dairy cattle studied using reaction norms. Acta Agric. Scand. Sect. Anim. Sci. 52:11-24. https://doi.org/10.1080/09064700252806380.

Liu, Z., M. E. Goddard, B. J. Hayes, F. Reinhardt, and R. Reents. 2016. Technical note: Equivalent genomic models with a residual polygenic effect. J. Dairy Sci. 99:2016-2025. https://doi.org/10 .3168/jds.2015-10394.
Lohuis, M. M., and J. C. M. Dekkers. 1998. Merits of borderless evaluations. Pages 169-172 in Proc. 6th World Congr. Genet. Appl. Livest. Prod. Sci., Armidale, Australia.

Lohuis, M. M., L. R. Schaeffer, and P. Rozzi. 1996. Developments in applying MACE to the conformation traits. Interbull Bull. 14:2633. https://journal.interbull.org/index.php/ib/article/view/324.

Makanjuola, B. O., F. Miglior, E. A. Abdalla, C. Maltecca, F. S. Schenkel, and C. F. Baes. 2020. Effect of genomic selection on rate of inbreeding and coancestry and effective population size of Holstein and Jersey cattle populations. J. Dairy Sci. 103:5183-5199. https://doi.org/10.3168/jds.2019-18013.

Mäntysaari, E. A., Z. Liu, and P. M. VanRaden. 2010. Interbull validation test for genomic evaluations. Interbull Bull. 41:17-21. https:// journal.interbull.org/index.php/ib/article/view/1134.

Mark, T., W. F. Fikse, U. Emanuelson, and J. Philipsson. 2002. Short communication: Effect of phantom parent grouping and properties of deregression for a low heritability trait. J. Dairy Sci. 85:23932395. https://doi.org/10.3168/jds.S0022-0302(02)74320-8.

Mark, T., and P. G. Sullivan. 2006. Multiple-trait multiple-country genetic evaluations for udder health traits. J. Dairy Sci. 89:48744885. https://doi.org/10.3168/jds.S0022-0302(06)72536-X.

Masuda, Y., P. M. VanRaden, I. Misztal, and T. J. Lawlor. 2018. Differing genetic trend estimates from traditional and genomic evaluations of genotyped animals as evidence of preselection bias in US Holsteins. J. Dairy Sci. 101:5194-5206. https://doi.org/10.3168/ jds.2017-13310.

Meuwissen, T. H. E., B. J. Hayes, and M. E. Goddard. 2001. Prediction of total genetic value using genome-wide dense marker maps. Genetics 157:1819-1829. https://doi.org/10.1093/genetics/157.4 .1819 .

Mrode, R. A. 2005. Best linear unbiased prediction of breeding value: Multivariate models. Pages 83-120 in Linear Models for the Prediction of Animal Breeding Values. 2nd ed. Wiley.

Nilforooshan, M. A. 2011. Multiple-trait multiple country genetic evaluation of fertility traits in dairy cattle. PhD thesis. Department of Animal Breeding and Genetics, Swedish Univ. Agric. Sci., Uppsala. https://pub.epsilon.slu.se/8094.

Nilforooshan, M. A. 2020. Application of single-step GBLUP in New Zealand Romney sheep. Anim. Prod. Sci. 60:1136-1144. https:// doi.org/10.1071/AN19315.

Nilforooshan, M. A., J. H. Jakobsen, W. F. Fikse, B. Berglund, and H. Jorjani. 2010a. Application of a multiple-trait, multiple-country genetic evaluation model for female fertility traits. J. Dairy Sci 93:5977-5986. https://doi.org/10.3168/jds.2010-3437.

Nilforooshan, M.A., J.H. Jakobsen, W.F. Fikse, B. Berglund, and H. Jorjani. 2014. Multiple-trait multiple country genetic evaluation of Holstein bulls for female fertility and milk production traits. Animal 8:887-894. https://doi.org/10.1017/S1751731114000895.

Nilforooshan, M. A., B. Zumbach, J. Jakobsen, A. Loberg, H. Jorjani, and J. Dürr. 2010b. Validation of national genomic evaluations. Interbull Bull. 42:56-61. https://journal.interbull.org/index.php/ $\mathrm{ib} /$ article/view/1158.

Pasman, E., and F. Reinhardt. 2002. Feasibility of international genetic evaluation for calving ease and stillbirth in Holstein. Interbull Bull. 29:49-54. https://journal.interbull.org/index.php/ib/article/ view $/ 732$.

Patry, C., H. Jorjani, and V. Ducrocq. 2013. Effects of a national genomic preselection on the international genetic evaluations. J. Dairy Sci. 96:3272-3284. https://doi.org/10.3168/jds.2011-4987.

Rekaya, R., K. A. Weigel, and D. Gianola. 2001. Application of a structural model for genetic covariances in international dairy sire evaluations. J. Dairy Sci. 84:1525-1530. https://doi.org/10.3168/ jds.S0022-0302(01)70186-5.

Rozzi, P., L. R. Schaeffer, E. B. Burnside, and W. Schlote. 1990. International evaluation of Holstein-Friesian dairy sires from three countries. Livest. Prod. Sci. 24:15-28. https://doi.org/10.1016/ 0301-6226(90)90028-5.

Ruhlmann, C., S. Janssens, J. Philipsson, E. Thorén-Hellsten, H. Crolly, K. Quinn, E. Manfredi, and A. Ricard. 2009. Genetic correlations between horse show jumping competition traits in five 
European countries. Livest. Sci. 122:234-240. https://doi.org/10 .1016/j.livsci.2008.09.006.

Schaeffer, L. R. 1985. Model for international evaluation of dairy sires. Livest. Prod. Sci. 12:105-115. https://doi.org/10.1016/0301 -6226(85)90084-3.

Schaeffer, L. R. 1994. Multiple-country comparison of dairy sires. J. Dairy Sci. 77:2671-2678. https://doi.org/10.3168/jds.S0022 -0302(94)77209-X.

Schaeffer, L. R. 2001. Multiple trait international bull comparisons. Livest. Prod. Sci. 69:145-153. https://doi.org/10.1016/S0301 $-6226(00) 00255-4$

Schaeffer, L. R. 2006. Strategy for applying genome-wide selection in dairy cattle. J. Anim. Breed. Genet. 123:218-223. https://doi.org/ 10.1111/j.1439-0388.2006.00595.x.

Schaeffer, L. R. 2018. Necessary changes to improve animal models. J. Anim. Breed. Genet. 135:124-131. https://doi.org/10.1111/jbg .12321.

Sigurdsson, A., and G. Banos. 1995. Dependent variables in international sire evaluations. Acta Agric. Scand. Sect. Anim. Sci. 45:209217. https://doi.org/10.1080/09064709509413079.

Stachowicz, K., M. Sargolzaei, F. Miglior, and F. S. Schenkel. 2011. Rates of inbreeding and genetic diversity in Canadian Holstein and Jersey cattle. J. Dairy Sci. 94:5160-5175. https://doi.org/10.3168/ jds.2010-3308.

Stolzman, M., H. Jasiorowski, Z. Reklewski, A. Zarnecki, and G. Kalinowska. 1988. Comparison of ten Friesian strains in Poland under field conditions. I. Strain comparison for growth rate. Livest. Prod. Sci. 18:217-237. https://doi.org/10.1016/0301-6226(88)90033-4.

Sullivan, P. G. 2013. GMACE reliability approximation. Interbull Bull. 47:1-4. https://journal.interbull.org/index.php/ib/article/ view/1283.

Sullivan, P. G. 2020. International genomic evaluation methods for dairy cattle. Advances in Breeding of Dairy Cattle, 1st ed. J. van der Werf and J. Pryce, ed. Burleigh Dodds Science Publishing.

Sullivan, P. G., E. Mäntysaari, G. DeJong, and H. Benhajali. 2019. Modifying MACE to accommodate genomic preselection effects. Interbull Bull. 55:77-80. https://journal.interbull.org/index.php/ $\mathrm{ib} /$ article/view/1484.

Sullivan, P. G., and P. M. VanRaden. 2009. Development of genomic GMACE. Interbull Bull. 40:157-160. https://journal.interbull.org/ index.php/ib/article/view/1104.

Sullivan, P. G., and J. W. Wilton. 2001. Multiple-trait MACE with a variable number of traits per country. Interbull Bull. 27:68-72. https://journal.interbull.org/index.php/ib/article/view/695.

Sullivan, P. G., J. W. Wilton, L. R. Schaeffer, G. J. Jansen, J. A. B. Robinson, and O. B. Allen. 2005. Genetic evaluation strategies for multiple traits and countries. Livest. Prod. Sci. 92:195-205. https: //doi.org/10.1016/j.livprodsci.2004.07.020.

Thorén Hellsten, E., H. Jorjani, and J. Philipsson. 2008. Connectedness among five European sport horse populations. Livest. Sci. 118:147-156. https://doi.org/10.1016/j.livsci.2007.12.028.

Thorén Hellsten, E., H. Jorjani, and J. Philipsson. 2009. Genetic correlations between similar traits in the Danish and Swedish Warmblood sport horse populations. Livest. Sci. 124:15-20. https://doi .org/10.1016/j.livsci.2008.11.021.

Tyrisevä, A.-M., W. F. Fikse, E. A. Mäntysaari, J. Jakobsen, G. P. Aamand, J. Dürr, and M. H. Lidauer. 2018. Validation of consistency of Mendelian sampling variance. J. Dairy Sci. 101:2187-2198. https://doi.org/10.3168/jds.2017-13255.

van der Linde, R., and G. de Jong. 2002. Feasibility of MACE for longevity traits. Interbull Bull. 29:55-60. https://journal.interbull .org/index.php/ib/article/view/733.

Van Tassell, C. P., T. P. L. Smith, L. K. Matukumalli, J. F. Taylor, R. D. Schnabel, C. T. Lawley, C. D. Haudenschild, S. S. Moore, W. C. Warren, and T. S. Sonstegard. 2008. SNP discovery and allele frequency estimation by deep sequencing of reduced representation libraries. Nat. Methods 5:247-252. https://doi.org/10.1038/ nmeth. 1185.

VanRaden, P. M. 2001. Methods to combine estimated breeding values obtained from separate sources. J. Dairy Sci. 84:E47-E55. https:/ /doi.org/10.3168/jds.S0022-0302(01)70196-8.
VanRaden, P. M. 2008. Efficient methods to compute genomic predictions. J. Dairy Sci. 91:4414-4423. https://doi.org/10.3168/jds .2007-0980.

VanRaden, P. M., and P. G. Sullivan. 2010. International genomic evaluation methods for dairy cattle. Genet. Sel. Evol. 42:7. https: //doi.org/10.1186/1297-9686-42-7.

VanRaden, P. M., and G. R. Wiggans. 1991. Derivation, calculation, and use of national animal model information. J. Dairy Sci. 74:2737-2746. https://doi.org/10.3168/jds.S0022-0302(91)78453 $-1$.

Wang, L., L. L. Janss, P. Madsen, J. Henshall, C.-H. Huang, D. Marois, S. Alemu, A. C. Sørensen, and J. Jensen. 2020. Effect of genomic selection and genotyping strategy on estimation of variance components in animal models using different relationship matrices. Genet. Sel. Evol. 52:31. https://doi.org/10.1186/s12711-020-00550 $-w$

Weigel, K. A. 1996. Use of correlated trait information to improve the accuracy of early predictions of breeding values for length of productive life. Interbull Bull. 12:125-135. https://journal.interbull .org/index.php/ib/article/view/259.

Weigel, K. A., and R. Rekaya. 2000. A multiple-trait herd cluster model for international dairy sire evaluation. J. Dairy Sci. 83:815-821. https://doi.org/10.3168/jds.S0022-0302(00)74944-7.

Wiggans, G. R., P. M. VanRaden, and R. L. Powell. 1992. A method for combining United States and Canadian bull evaluations. J. Dairy Sci. 75:2834-2839. https://doi.org/10.3168/jds.S0022 -0302(92)78046-1.

Wilmink, J. B. M., A. Meijering, and B. Engel. 1986. Conversion of breeding values for milk from foreign populations. Livest. Prod. Sci. 14:223-229. https://doi.org/10.1016/0301-6226(86)90081-3.

Worede, G.M., F. Forabosco, B. Zumbach, V. Palucci, and H. Jorjani. 2013. Evaluation of genetic variation in the international Brown Swiss population. Animal 7:1060-1066. https://doi.org/10.1017/ S1751731113000281.

Zarnecki, A., J. Jamrozik, and H. D. Norman. 1991. Comparison of ten Friesian strains in Poland for yield traits from first three parities. J. Dairy Sci. 74:2303-2308. https://doi.org/10.3168/jds.S0022 $-0302(91) 78403-8$

\section{ORCIDS}

M. A. Nilforooshan (®) https://orcid.org/0000-0003-0339-5442

H. Jorjani $\odot$ https://orcid.org/0000-0003-3375-3263

\section{APPENDIX 1}

Method of Jairath et al. (1998), with corrections and simplifications to the original paper, for the deregression of national proofs from country $i$. (Notations are defined in the article.)

Given $\mathbf{a}_{i}$ [the vector of sires' genetic evaluations $\left(c_{i} \mathbf{1}\right.$ $\left.\left.+\mathbf{Q g}_{i}+\mathbf{s}_{i}\right)\right]$, matrices $\mathbf{A}^{-1}$ and $\mathbf{R}_{i}^{-1}$, and $\mathrm{h}_{i}^{2}$, these steps are followed to compute the vector of deregressed proofs $\left(\mathbf{y}_{i}\right)$ :

1. $c_{i}=0$

2. $\left(\mathbf{Q g}_{i}+\mathbf{s}_{i}\right)=\mathbf{a}_{i}-c_{i} \mathbf{1}$

3. $\left[\begin{array}{l}\mathbf{p}_{i} \\ \mathbf{g}_{i}\end{array}\right]=-\left[\begin{array}{ll}\mathbf{A}^{p p} & \mathbf{A}^{p g} \\ \mathbf{A}^{g p} & \mathbf{A}^{g g}\end{array}\right]^{-1}\left[\begin{array}{l}\mathbf{A}^{p s} \\ \mathbf{A}^{g s}\end{array}\right]\left(\mathbf{Q g}_{i}+\mathbf{s}_{i}\right)$

4. $\left(\mathbf{R}_{i}^{-1} \mathbf{y}_{i}\right)=\mathbf{R}_{i}^{-1} \mathbf{1} c_{i}+\left(\mathbf{R}_{i}^{-1}+\mathbf{A}^{\mathrm{ss}} k_{i}\right)\left(\mathbf{Q g}_{i}+\mathbf{s}_{i}\right)+$ $\mathbf{A}^{s p} \mathbf{p}_{i} k_{i}+\mathbf{A}^{s g} \mathbf{g}_{i} k_{i}$

5. $c_{i}=\mathbf{1}^{\prime}\left[\left(\mathbf{R}_{i}^{-1} \mathbf{y}_{i}\right)-\mathbf{R}_{i}^{-1}\left(\mathbf{Q g}_{i}+\mathbf{s}_{i}\right)\right] / \mathbf{1}^{\prime} \mathbf{R}_{i}^{-1} \mathbf{1}$ 
6. If convergence is not met, go to step 2

7. $\mathbf{y}_{i}=\mathbf{R}_{i}\left(\mathbf{R}_{i}^{-1} \mathbf{y}_{i}\right)$

Step 3 involves solving rows 3 and 4, step 4 involves solving row 2, and step 5 involves solving row 1 of Eq. [7]. Jairath et al. (1998) did not mention how the convergence is met (step 6). One option is the relative difference between solutions from consecutive iterations being smaller than a small positive value $(\varepsilon)$ :

$$
\sqrt{\frac{\left[\left(\mathbf{R}^{-1} \mathbf{y}\right)_{j}-\left(\mathbf{R}^{-1} \mathbf{y}\right)_{j-1}\right]\left[\left(\mathbf{R}^{-1} \mathbf{y}\right)_{j}-\left(\mathbf{R}^{-1} \mathbf{y}\right)_{j-1}\right]}{\left(\mathbf{R}^{-1} \mathbf{y}\right)_{j}^{\prime}\left(\mathbf{R}^{-1} \mathbf{y}\right)_{j}}}<\varepsilon .
$$

\section{APPENDIX 2}

Method of Sullivan and Wilton (2001) for the derivation of multi-trait independent EDC factors. (Notations are defined in the article.)
The diagonal matrix, $\dot{\mathbf{D}}_{i k}^{-1}$, is computed for bull $k$ in country $i$, where $\varepsilon$ is a small positive value for the convergence criterion, $\mathbf{D}_{i k}^{-1}$ is the corresponding matrix used in MACE, and $G_{j j}, R_{j j}$, and $B_{j j}$ are the $j$ th diagonal elements (for the jth of $t$ number of traits) of $\mathbf{G}_{i}, \mathbf{R}_{i}$, and $\mathbf{B}$, respectively:

1. $l=0$

2. For $j$ in 1 to $t$ :

2.1. $\dot{D}_{i j k}^{-1(l)}=D_{i j k}^{-1}$

3. $\mathbf{B}^{(l)}=\left\{\operatorname{diag}\left[\operatorname{diag}\left(\dot{\mathbf{D}}_{i k}^{-1(l)}\right) / \operatorname{diag}\left(\mathbf{R}_{i}\right)\right]+\mathbf{G}_{i}^{-1}\right\}^{-1}$

4. For $j$ in 1 to $t$ :

4.1. $e_{j}^{(l)}=D_{i j k}^{-1}-R_{j j}\left(G_{j j}-B_{j j}^{(l)}\right) /\left(G_{j j} B_{j j}^{(l)}\right)$

4.2. $\dot{D}_{i j k}^{-1(l+1)}=\dot{D}_{i j k}^{-1(l)}+e_{j}^{(l)} \times$ relax

5. $l=l+1$

6. If $\max \left(\mathbf{e}^{(l-1)}\right) \geq \varepsilon$, go to step 3

7. Return $\dot{\mathbf{D}}_{i k}^{-1(l)}$ 